\title{
Dynamics and Control of Tethered Formation Flight Spacecraft Using the SPHERES Testbed
}

\author{
Soon-Jo Chung* Edmund M. Kong ${ }^{\dagger}$ \\ David W. Miller $\ddagger$ \\ Space Systems Laboratory, Massachusetts Institute of Technology, MA 02139, USA
}

\begin{abstract}
This paper elaborates on the theory and experiment of controlling tethered spacecraft formation without depending on thrusters. In dealing with such underactuated systems, much emphasis is placed on complete decentralization of the control and estimation algorithms in order to reduce the dimensionality and complication. The nonlinear equations of motions of multi-vehicle tethered spacecraft are derived by Lagrange's equations. Decentralization is then realized by the diagonalization technique and its stability is proven by contraction theory. The preliminary analysis predicts unstable dynamics depending on the direction of the tether motor. The controllability analysis indicates that both array resizing and spin-up are fully controllable only by the reaction wheels and the tether motor, thereby eliminating the need for thrusters. Based upon this analysis, gain-scheduling LQR controllers and nonlinear controllers by feedback linearization have been successfully implemented into the tethered SPHERES testbed, and tested at the NASA MSFCs flat floor facility using two and three SPHERES configurations. The relative sensing mechanism employing the ultrasound ranging system and the inertial gyro is also described.
\end{abstract}

\section{Introduction}

$\mathrm{T}$ HE quest for finer angular resolution in astronomy inevitably leads to larger apertures since the optical resolution is proportional to the wavelength over the diameter of a circular aperture. Unfortunately, the primary mirror diameter for space telescopes is limited by volume and mass constraints of current launch vehicles (ca. 4 to $5 \mathrm{~m}$ ) as well as the scaling laws of manufacturing cost. ${ }^{1}$ In order to overcome this difficulty, break-through technologies such as a Separated Spacecraft Interferometer (SSI) are being studied for NASA's Terrestrial Planet Finder (TPF) mission. ${ }^{2}$ Other benefits to using such a system include array reconfigurability and upgradability. Another formation flight concept that has been considered for a SSI system is the use of tether. To image a target, measurements must be made in all directions orthogonal to the line-of-sight of the array. The balance between using a Structurally Connected Interferometer (SCI), which allows for very limited baseline changes, and a SSI system where the usage of propellant can be prohibitively expensive, seems to be using a tethered system. Such a system is currently being considered for NASA's Submillimeter Probe of the Evolution of Cosmic Structure (SPECS) mission. ${ }^{3}$ The SPECS mission attempts to address fundamental questions about our universe (e.g. How did the first stars form from primordial material, and the first galaxies from pre-galactic structures? How did the galaxies evolve over time?) by looking at the sky at sub-millimeter wavelength. One mission concept is to use a Tethered Spacecraft Interferometer (TSI) system to maneuver the sub-apertures out to separations of a kilometer, thereby achieving very high resolution. Since power, maneuvering loads and data can be supported by the tether, these typical spacecraft functions are not required on the maneuvering vehicles. This reduces replication of sub-systems across the various sub-apertures and eliminates the need for propellant. Furthermore, the mass per unit length of the tether is much smaller than that of a deployed truss making it much more mass-efficient, particularly for long baselines. However, all of these benefits are lost if the control needed to achieve the precision required by the array proves to be too complex. It is expected that vibratory motion, consisting of compound pendulum

*Doctoral Student, Department of Aeronautics and Astronautics, and AIAA Student Member, sjchung@mit.edu

${ }^{\dagger}$ Research Scientist, Department of Aeronautics and Astronautics, and AIAA Member, mckong@mit.edu

${ }^{\ddagger}$ Associate Professor, Department of Aeronautics and Astronautics, and AIAA Senior Member, millerd@mit.edu 
mode of the satellite bus and tether violin modes, will be observed during operations of a TSI. Highly maneuverable spacecraft are particularly problematic since beam control in the optics system will need to be maintained to the requisite precision while thrusters fire, tethers vibrate, and reaction wheels change momentum. These introduce harsh disturbances that necessitate the coupling of attitude and optical control. This paper describes efforts made to facilitate the development of dynamics and control work required for a future space tethered system.

\section{A. Research Objective}

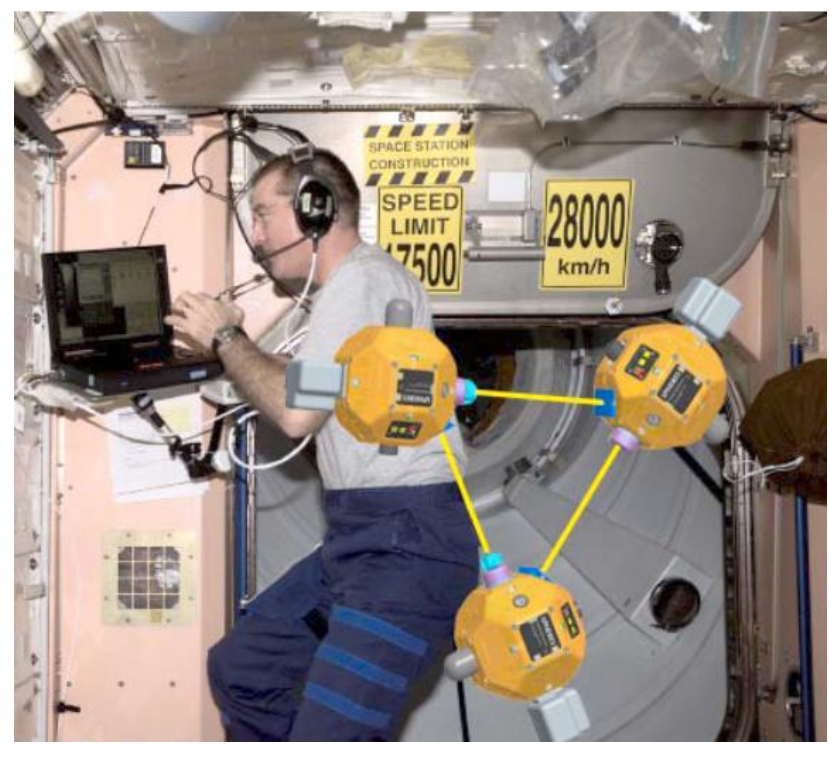

Figure 1. Artist's concept of tethered formation flying SPHERES in ISS cell

Another goal is to develop sophisticated decentralized control and estimation algorithms for the tethered system eliminating the need for satellite communications. Decentralized means that each measurement or estimation process from measurement must not involve exchange of information with adjacent satellites. Relative means that measured quantity is formulated with respect to a local frame of individual SPHERES. The decentralized controller along with the decentralized estimator will enable a simple independent control of each satellite without the need for exchanging individual states information. This will significantly simplify both control algorithm and hardware implementation with lesser degree of complication as well as eliminating communication delays. This distributed architecture also avoids a fatal flaw of a centralized control system: if the chief member malfunctions or stops operation, the whole formation could be lost. ${ }^{4}$ The decentralized scheme is realized by the decoupling technique and contraction theory introduced in Section IV.

A relative attitude estimation technique is also employed as opposed to the absolute attitude estimation. In deep space, absolute attitude might be available via star-trackers but availability of absolute positions will be very limited. The relative sensing system is especially beneficial for the deep space missions, where accurate absolute position measurements like GPS are not available. ${ }^{5}$ Relative sensing mechanism is realized based upon the dynamics modeling in the previous section. Note that all the degrees of freedom defined in section III are relative states (e.g. bearing angle, $\phi$ ).

\section{B. Previous Work}

The Synchronized Position Hold Engage and Reorient Experimental Satellites (SPHERES) ${ }^{6}$ testbed will be the first satellite testbed fully exploiting the dynamics of tethered satellites under various formation flight configurations. It is also a fully 3-D operational satellite with sophisticated sensors and actuators as a moderepresentative of real world spacecraft in orbit even though we focus on 2-D flat floor experiments in this paper. A notable previous work on experiments of two tethered satellites is the Ph.D thesis by Motley. ${ }^{7}$ 
A recent paper also describes the tethered testbed ${ }^{9}$ with a triangular configuration. The classic reference for tether dynamics is a book by Beletsky, et al. ${ }^{8}$ There are a lot of technical papers on the dynamics of tether in space. ${ }^{10-12}$ Some recent papers discuss the elasticity and vibration of the tethers. ${ }^{12,13}$ Compared to the early literature focused on the two-body dynamics for the tether retrieval and momentum exchange purposes, more recent work investigates the dynamics of a three-body inline configuration ${ }^{16}$ and a triangular configuration. ${ }^{17}$ This SPHERES project studies both configurations for the SPECS mission. Development of nonlinear controller was also performed for the SPECS mission by Mischa. ${ }^{14,15}$

\section{Development of SPHERES Hardware}

The MIT SPHERES testbed provides a vehicle to demonstrate and validate formation flight and docking technologies for use in missions such as TPF and Orbital Express. Among the technologies that are actively under study by SPHERES are space interferometry, cluster reconfiguration, and mission re-supply. Many of these techniques can only be tested in simulation or with expensive and risky flight projects. Currently, there are no on-orbit resources suitable for the validation of general simulation results, and most space missions do not push the limits of performance due to the high risk associated with flying unproven algorithms. The SPHERES testbed is an inexpensive and risk-tolerant laboratory for the validation of distributed spacecraft control, estimation, and autonomy algorithms. It fills the gap between the flexibility, risk-tolerance, and uncertainty of simulation-based research and the inflexibility, expense, and credibility expected from future space flight missions. The SPHERES testbed was developed as part of the ongoing research initiatives of the MIT Space Systems Laboratory (MIT-SSL) that utilize the space environment provided by the space shuttle and International Space Station (ISS) to validate dynamics and control algorithms.

\section{A. Tethered SPHERES Overview}
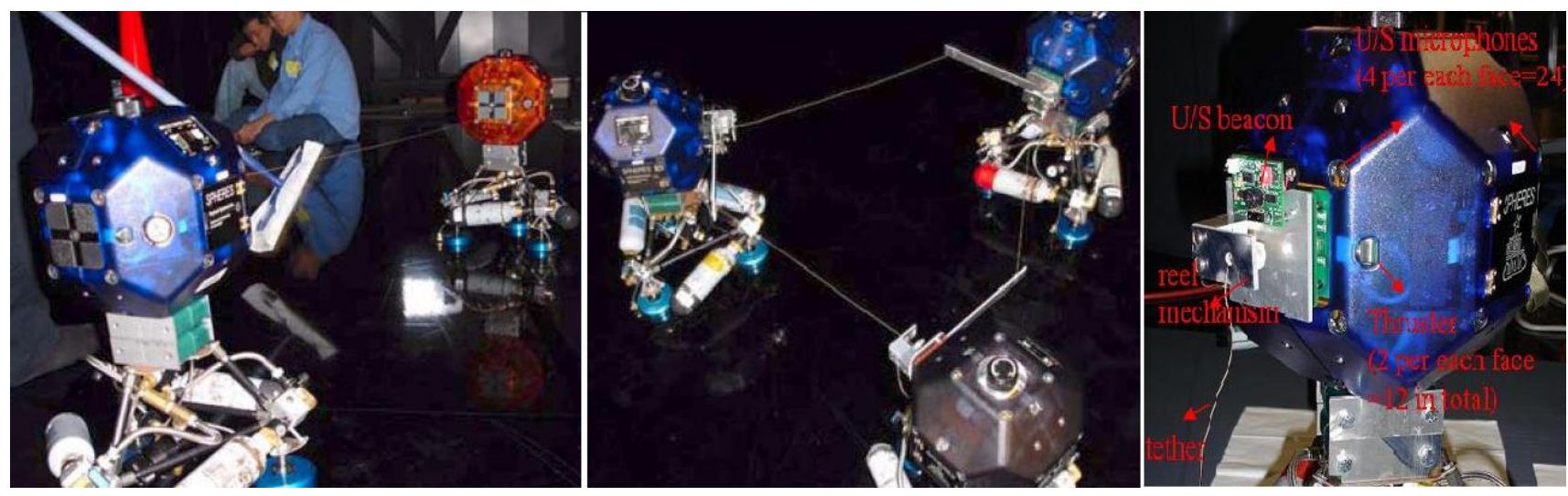

Figure 2. Tethered SPHERES satellites on the NASA MSFC flat floor

The basic setup of the SPHERES testbed consists of a number of autonomous free-flyers satellites, a laptop computer that serves as a ground station, and small beacons that form the Position and Attitude Determination System (PADS). SPHERES has three main operational environments: simulation, flat floor facility (at MIT and NASA Marshall Space Flight Center), and 3-D environment (inside the ISS and NASA's KC-135 flight). The simulation allows the operation of up to three satellite models in any standard PC running Windows operating system. The configuration at the SSL facility consists of multiple operational flight-qualified SPHERES satellites, a metrology setup optimized for 2-D operations, and a metrology setup designed for 3-D operations. The final ISS configuration will consist of three tethered satellites and a 3-D metrology setup (See figure. 1). Both MIT-SSL and ISS setups use a laptop computer to represent a ground control station; through the laptop, the user runs tests and collects telemetry data for future analysis. The laptop computer utilizes a custom communications device to control the satellites and store all the data. Different interfaces were developed for the ISS and ground operations; the ground interface minimizes the time between tests, while the ISS operations clearly steps through the operation procedures to ensure correct 
tests are being implemented.

\section{B. SPHERES Satellites}

The individual self-contained satellites (figure. 2) have the ability to maneuver in up to six degrees of freedom, to communicate with each other and with the laptop control station, and to identify their position with respect to each other and to the experiment reference frame. The diameter of a single SPHERES is $0.25 \mathrm{~m}$, and the mass is $4.0 \mathrm{~kg}$. The satellites are propelled by a cold-gas thruster system which uses carbon dioxide as propellant. The CO2 propellant is stored in liquid form at 860 psig; a regulator reduces the pressure to 35 psig. Twelve thrusters are positioned to provide controllability in all six degrees of freedom, enabling both torque and translation control. Each thruster assembly consists of a solenoid-actuated microvalve with machined nozzles. Depending upon the tests of interest, a single tank provides approximately 30 minutes of active operation. After each test session, a tank can be left in the system partially full, for use at a later time, or be replaced with a new tank. The PADS provides metrology information to the satellites in real-time. Since no global metrology system like GPS is actually available in deep space missions, the tethered SPHERES system utilizes a relative metrology system using four ultrasound receivers on the lineof-sight face and the on-board beacon of the adjacent SPHERES (see figure. 2). The relative metrology system is a pseudo-GPS ranging system that uses ultrasonic time-of-flight measurements from the target onboard beacon to the ultrasonic microphones distributed on the surface of each satellite. These time-of-flight measurements are converted to ranges and are then used to derive position and attitude with respect to the reference frame using a series of Extended Kalman Filters(EKF). A detailed description on the relative metrology system will follow in section V. A Texas Instruments C6701 Digital Signal Processor provides the computational power. The ability of the C6701 to provide up to 1.0 GFLOPS provides significant processing power to prevent being the limiting factor in the performance of the system. A FLASH memory size of 224 $\mathrm{KB}$ allows software re-configuration of the full operating system, ensuring that multiple investigators are supported while the system is in the ISS. The power system utilized onboard the ISS consists of packs of AA alkaline batteries while NiMH rechargeable packs are used on the ground facility. The packs provide each satellite with approximately two hours of operation; once a pack is consumed, it can be easily replaced. Each SPHERES satellite uses two separate frequency communications channels operating at $57.6 \mathrm{kbps}$ each. One channel is used for satellite-to-satellite communications; the other channel enables satellite-to-laptop communications. Both channels are bi-directional; however, the communication hardware is half-duplex, meaning that only one unit can transmit at a time.

\section{Modification for Tethered Formation}

The tether package can be divided into three major components: the tether deployment and retraction mechanism with tether tension sensors; latch plate using velcro; and momentum wheel package. Prior to implementing the reaction wheels, torque generated by diagonal thrusters is employed in the experiments presented in this paper. The reel in/out mechanism is used to control the tethers during extension and contraction. It allows the tether to smoothly and orderly maintain a tension between satellites. The tether from the deployment mechanism is connected to the latch plate on the other satellite. The momentum wheel package, which consists of 3 wheels, will be located on the opposite side of the tether mechanism to counter-balance the weight introduced by the tether mechanism. Note that both the tether deployment mechanism and momentum wheel assembly are controlled through the satellite's expansion port and are designed to be modular where one design fits all three satellites. The relative metrology system provides measure of the separation distance for feedback control. The completion of this work will result in a flight rated tether mechanism ready for integration onboard the Shuttle or ISS. With this hardware, dynamics and control work are then be studied and demonstrated on a 2-D flat floor facility prior to testing in a more realistic 3-D environment, onboard the ISS.

\section{Nonlinear Dynamics Modeling}

\section{A. Assumptions}

For the sake of simplicity, the dynamics relevant only to the 2-D flat floor test is formulated here before the full 3-D equations of motions accounting for out-of-plane motions are investigated. Figure 3 shows a single 
SPHERES revolving about the center of the fixed inertial axes X-Y. The rotating axes, $\mathrm{x}-\mathrm{y}$ are chosen such that the $\mathrm{y}$ direction always coincides with the direction of increasing $\theta$ while the $\mathrm{x}$ direction is aligned with the tether. The SPHERES is also exhibiting a compound pendulum rotation with respect to $\mathrm{A}$. The force and torque exerted on the Center of Mass (CM) are drawn in black. $T$ is the tension force of the tether, $F_{x}$ and $F_{y}$ are the x-y directional forces due to thruster firing, and $u$ is the torque exerted on the CM (point B), e.g torque by RWA.

The following assumptions are made regarding the tether wire. The tether is massless and inextensible, however it is planned to account for the effect of the elasticity of the tether in the near future. Additionally, the array is assumed to always rotate at a certain angular rate so the tether is taut and straight at all times. A non-zero angular rotation is a realistic assumption since tethered interferometers will attempt to fill a full $\mathrm{u}-\mathrm{v}$ coverage by rotation. However, it was observed from the experiments that the assumption of tautness is no longer valid when the satellites bounce off the tether by highimpedance actuation. We plan to mitigate this effect by adding some flexibility to the tether (e.g. spring). We also assume a constant reel-in or reel-out speed $i$. The speed of the tether motor can be easily pre-defined in the control code and does not constitute the system states. The gravity term is omitted on the assumption that a tethered formation array will operate in the very weak gravity field such as the Lagrangian point L2. Control-wise, any additional un-modeled gravity is regarded as

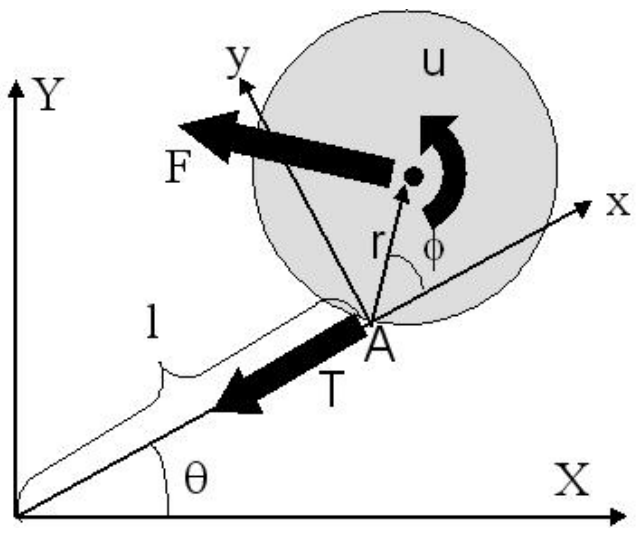
a low-frequency disturbance to the system.

\section{B. Derivation of Nonlinear Equations}

In order to reduce the complexity of trigonometric functions (sine and cosine), the velocity and acceleration will be expressed in the small $\mathrm{x}-\mathrm{y}$ rotating frame and the corresponding unit directional vectors are $e_{x}$ and $e_{y}$.

The velocity of $\mathrm{B}$ is characterized as

$$
v_{B}=[i-r \sin \phi(\dot{\theta}+\dot{\phi})] e_{x}+[r \cos \phi(\dot{\theta}+\dot{\phi})+l \dot{\theta}] e_{y}
$$

The relationship between the absolute and relative acceleration is obtained by differentiating the relative velocity equation to get

$$
a_{B}=a_{A}+\ddot{\theta} e_{z} \times r_{B / A}+\dot{\theta} e_{z} \times\left(\dot{\theta} e_{z} \times r_{B / A}\right)+2 \dot{\theta} e_{z} \times v_{B / A}+a_{B / A}
$$

Since the SPHERES is rotating in the x-y frame that is revolving around the center of the fixed inertial $\mathrm{X}-\mathrm{Y}$ frame, we are observing the coriolis term $\left(2 \dot{\theta} e_{z} \times v_{B / A}\right)$ as well as the centrifugal force $\left(\dot{\theta} e_{z} \times\left(\dot{\theta} e_{z} \times r_{B / A}\right)\right)$.

Each term in Eq. (2) is calculated:

$$
\begin{gathered}
a_{A}=\left(-l \dot{\theta}^{2}+\ddot{l}\right) e x+(l \ddot{\theta}+2 i \dot{\theta}) e_{y} \\
\ddot{\theta} e_{z} \times r_{B / A}=-r \sin \phi \ddot{\theta} e_{x}+r \cos \phi \ddot{\theta} e_{y} \\
\dot{\theta} e_{z} \times\left(\dot{\theta} e_{z} \times r_{B / A}\right)=-r \cos \phi \dot{\theta}^{2} e_{x}-r \sin \phi \dot{\theta}^{2} e_{y} \\
2 \dot{\theta} e_{z} \times v_{B / A}=-2 r \cos \phi \dot{\theta} \dot{\phi} e_{x}-2 r \sin \phi \dot{\theta} \dot{\phi} e_{y} \\
a_{B / A}=\left(-r \ddot{\phi} \sin \phi-r \dot{\phi}^{2} \cos \phi\right) e_{x}+\left(r \ddot{\phi} \cos \phi-r \dot{\phi}^{2} \sin \phi\right) e_{y}
\end{gathered}
$$

Let's assume for now the tether length is fixed. This means that $a_{A}=\left(-l \dot{\theta}^{2}+\ddot{l}\right) e x+(l \ddot{\theta}+2 i \dot{\theta}) e_{y}$ is reduced to $a_{A}=\left(-l \dot{\theta}^{2}\right) e x+(l \ddot{\theta}) e_{y}$ Then, Eq.(2) becomes

$$
\begin{array}{r}
a_{B}=e_{x}\left[-l \dot{\theta}^{2}-r \sin \phi \ddot{\theta}-r \cos \phi \dot{\theta}^{2}-2 r \cos \phi \dot{\theta} \dot{\phi}-r \ddot{\phi} \sin \phi-r \dot{\phi}^{2} \cos \phi\right]+ \\
e_{y}\left[(l+r \cos \phi) \ddot{\theta}-r \sin \phi \dot{\theta}^{2}-2 r \sin \phi \dot{\theta} \dot{\phi}+r \ddot{\phi} \cos \phi-r \dot{\phi}^{2} \sin \phi\right]
\end{array}
$$


The equations of the tethered system is derived using Eq. (4):

$$
\begin{array}{r}
\sum F_{e_{x}}=-F_{x}-T=-F \sin \phi-T=m a_{x} \\
\sum F_{e_{y}}=F_{y}=F \cos \phi=m a_{y} \\
\sum M_{G}=-T r \sin \phi+u=I_{G}(\ddot{\theta}+\ddot{\phi})
\end{array}
$$

where $M_{G}$ is the moment around $\mathrm{CM}$ and $I_{G}$ denotes the moment of inertia around CM. $a_{x}$ and $a_{y}$ are the $\mathrm{x}, \mathrm{y}$ acceleration components of Eq. (4) respectively.

$T$ can be eliminated and the following differential equation is obtained,

$$
[M(\phi)]\left(\begin{array}{c}
\ddot{\theta} \\
\ddot{\phi}
\end{array}\right)+[C(\phi, \dot{\theta}, \dot{\phi})]=\left(\begin{array}{c}
F \cos \phi \\
F r+u
\end{array}\right)
$$

where $[M(\phi)]=\left[\begin{array}{cc}m l+m r \cos \phi & m r \cos \phi \\ I_{G}+m r^{2}+m r l \cos \phi & I_{G}+m r^{2}\end{array}\right]$ and $[C(\phi, \dot{\theta}, \dot{\phi})]=\left[\begin{array}{c}-2 m r \sin \phi \dot{\theta} \dot{\phi}-m r \sin \phi \dot{\theta}^{2}-m r \sin \phi \dot{\phi}^{2} \\ m r l \sin \phi \dot{\theta}^{2}\end{array}\right]=\left[\begin{array}{c}-m r \sin \phi(\dot{\theta}+\dot{\phi})^{2} \\ m r l \sin \phi \dot{\theta}^{2}\end{array}\right]$

When the tether motor reels in or out at a constant speed $(i=$ constant), the force term in Eq. (6) is characterized as:

$$
\left(\begin{array}{c}
F \\
u
\end{array}\right) \Rightarrow\left(\begin{array}{c}
F-2 m i \dot{\theta} \\
u
\end{array}\right)
$$

The equations of the motion can be also derived by exploiting the technology developed for multi-link robot kinematics or Lagrange's equation. Those equations are simplified assuming the mass of the first link is zero (therefore, the inertia of moment is zero as well). Then, the governing equation of motion becomes

$$
[M(\phi)]\left(\begin{array}{c}
\ddot{\theta} \\
\ddot{\phi}
\end{array}\right)+[C(\phi, \dot{\theta}, \dot{\phi})]=\left(\begin{array}{l}
\tau_{1} \\
\tau_{2}
\end{array}\right)
$$

where $[M(\phi)]=\left[\begin{array}{cc}I_{G}+m r^{2}+m l^{2}+2 m r l \cos \phi & I_{G}+m r^{2}+m r l \cos \phi \\ I_{G}+m r^{2}+m r l \cos \phi & I_{G}+m r^{2}\end{array}\right]$ and $[C(\phi, \dot{\theta}, \dot{\phi})]=\left[\begin{array}{c}-2 m r l \sin \phi \dot{\theta} \dot{\phi}-m r l \sin \phi \dot{\phi}^{2} \\ m r l \sin \phi \dot{\theta}^{2}\end{array}\right]$

Compared to Eq.(6), Eq.(8) has more simpler form of $C(\phi, \dot{\theta}, \dot{\phi})$ function and the inertia matrix, $M$ is now symmetric. The torque $\tau_{1}$ and $\tau_{1}$ are now applied to the joints $\mathrm{O}$ (origin of $\mathrm{X}-\mathrm{Y}$ ) and $\mathrm{A}$ (origin of $\mathrm{x}-\mathrm{y}$ ).

It is shown Eq.(6) and Eq.(8) are essentially the same equations since 1st row times $l$ plus 2 nd row of Eq. (6) results in the first equation of Eq. (8).

So the external force(or torque) terms can be matched like the following:

$$
\left(\begin{array}{l}
\tau_{1} \\
\tau_{2}
\end{array}\right)=\left[\begin{array}{cc}
r+l \cos \phi & 1 \\
r & 1
\end{array}\right]\left(\begin{array}{l}
F \\
u
\end{array}\right)
$$

\section{Linearization and Pendulum Mode Frequency}

We linearize Eq. (8) about $\dot{\theta}=\omega$, and $\dot{\phi}, \phi=0$.

Each term can be linearized as the following: $m r l \sin \phi \dot{\theta}^{2} \approx m r l \omega^{2} \phi, m r l \sin \phi \dot{\phi}^{2} \approx 0, m r l \sin \phi \dot{\theta} \dot{\phi} \approx$ $0, \cos \phi \approx 1$

The linearized equation of the motion is presented:

$$
\left[\begin{array}{cc}
I_{G}+m(r+l)^{2} & I_{G}+m r(r+l) \\
I_{G}+m r(r+l) & I_{G}+m r^{2}
\end{array}\right]\left(\begin{array}{c}
\ddot{\theta} \\
\ddot{\phi}
\end{array}\right)+\left[\begin{array}{cc}
0 & 0 \\
0 & m r l \omega^{2}
\end{array}\right]\left(\begin{array}{c}
\theta \\
\phi
\end{array}\right)=\left(\begin{array}{c}
\tau_{1} \\
\tau_{2}
\end{array}\right)
$$


Similarly, we can linearize Eq. (6) and transformation between the two system equations is easily performed using Eq. (9).

It is observed that nonzero rotational rate, $\omega$ added a potential term to the dynamics even though there is no gravitational force in the model. This nonzero artificial potential energy induced by the centrifugal force of array rotation plays a crucial role in making the system controllable and stable. This is especially true for underactuated systems with $(F=0)$.

A nice property about this linearized equation (10) is that we can decouple the equation of $\phi$ from that of $\theta$. The first equation is merely the dynamics of a rigid body mode of $\theta$ (rotation about the center of X-Y).

Since the inertia matrix is invertible, we multiply both sides of Eq. (10) by the inverse of $M$ matrix and use the linearized relationship of Eq. (9):

$$
\left(\begin{array}{c}
\ddot{\theta} \\
\ddot{\phi}
\end{array}\right)+\left[\begin{array}{cc}
0 & -\frac{r \omega^{2}\left(I_{G}+m r(r+l)\right)}{l_{G}} \\
0 & \frac{r \omega^{2}\left(I_{G}+m(r+l)^{2}\right)}{l I_{G}}
\end{array}\right]\left(\begin{array}{l}
\theta \\
\phi
\end{array}\right)=\left[\begin{array}{cc}
\frac{1}{m l} & -\frac{r}{I_{G} l} \\
-\frac{1}{m l} & \frac{r+l}{I_{G} l}
\end{array}\right]\left(\begin{array}{l}
F \\
u
\end{array}\right)
$$

The second-order nonlinear equation of motion of $\phi$ from the second line of (11) becomes:

$$
\ddot{\phi}+\omega_{\phi}^{2} \phi=-\frac{1}{m l} F_{y}+\frac{r+l}{I_{G} l} u
$$

where $\omega_{\phi}$ is the frequency of the pendular libration mode:

$$
\omega_{\phi}=\sqrt{\frac{r\left(I_{G}+m(r+l)^{2}\right)}{l I_{G}}} \omega[\mathrm{rad} / \mathrm{s}]
$$

The actual raw gyro data from a single tethered SPHERES exhibit a high frequency oscillation (the pendulum mode) and the DC component (a rigid body mode of a certain rotational rate). The frequency obtained by Eq. (13) correctly predicted the actual frequency obtained from the gyro data with a less than 5 percent error.

\section{Controllability of LTI System}

The Linear Time Invariant (LTI) system is presented from Eq. (11) as the following.

$$
\frac{d}{d t}\left(\begin{array}{c}
\theta \\
\phi \\
\dot{\theta} \\
\dot{\phi}
\end{array}\right)=\left[\begin{array}{cccc}
0 & 0 & 1 & 0 \\
0 & 0 & 0 & 1 \\
0 & \frac{r \omega^{2}\left(I_{G}+m r(r+l)\right)}{l I_{G}} & 0 & 0 \\
0 & -\frac{r \omega^{2}\left(I_{G}+m(r+l)^{2}\right)}{l I_{G}} & 0 & 0
\end{array}\right]\left(\begin{array}{c}
\theta \\
\phi \\
\dot{\theta} \\
\dot{\phi}
\end{array}\right)+\left[\begin{array}{cc}
0 & 0 \\
0 & 0 \\
\frac{1}{m l} & -\frac{r}{I_{G} l} \\
-\frac{1}{m l} & \frac{r+l}{I_{G} l}
\end{array}\right]\left(\begin{array}{c}
F \\
u
\end{array}\right)
$$

where the first matrix of the righthand side is the $4 \times 4$ system matrix $A$ and the second is the $4 \times 2$ input matrix $B$. Note that $\theta$ is easily eliminated by removing the first column and the first of row of $A$ matrix, thereby reducing the dimension to three.

Let's check if the system is really controllable around nominal points by calculating controllability matrix.

$$
\left[\begin{array}{llll}
B & A B & A^{2} B & A^{3} B
\end{array}\right]=\left[\begin{array}{cccc}
0 & 0 & \frac{1}{m l} & -\frac{r}{I_{G} l} \\
0 & 0 & -\frac{1}{m l} & \frac{r+l}{I_{G} l} \\
\frac{1}{m l} & -\frac{r}{I_{G} l} & 0 & 0 \\
-\frac{1}{m l} & \frac{r+l}{I_{G} l} & 0 & 0
\end{array}\right]
$$

Where the calculation of $A^{2} B$ and $A^{3} B$ is omitted since the first two matrices result in the full rank $(n=4)$ and the rest of them are redundant (dependent). So the system is fully controllable with $u$ (Control torque on CM by RWA or coupled thruster firing) and $F$ (linear thruster firing).

In fact, all the states $(\theta, \phi, \dot{\theta}, \dot{\phi})$ are controllable only by the torque generating actuator $u$ (e.g. RWA). The controllability matrix using the second column of $B$ matrix in Eq. (14) becomes: 


$$
\left[\begin{array}{llll}
B_{2} & A B_{2} & A^{2} B_{2} & A^{3} B_{2}
\end{array}\right]=\left[\begin{array}{cccc}
0 & -\frac{r}{I_{G} l} & 0 & \frac{r(r+l) \omega^{2}\left(I_{G}+m r(r+l)\right)}{l^{2} I_{G}{ }^{2}} \\
0 & \frac{r+l}{I_{G} l} & 0 & -\frac{r(r+l) \omega^{2}\left(I_{G}+m(r+l)^{2}\right)}{l^{2} I_{G}{ }^{2}} \\
-\frac{r}{I_{G} l} & 0 & \frac{r(r+l) \omega^{2}\left(I_{G}+m r(r+l)\right)}{l^{2} I_{G}{ }^{2}} & 0 \\
\frac{r+l}{I_{G} l} & 0 & -\frac{r(r+l) \omega^{2}\left(I_{G}+m(r+l)^{2}\right)}{l^{2} I_{G}{ }^{2}} & 0
\end{array}\right]
$$

This is a full rank $(n=4)$ matrix for any nonzero $\omega$ and tether length, $l$. Its implication to the future tethered systems is enormous: the tethered satellite systems will be able to spin up and re-size the array on the plane of the array without the use of propellant consumable when operating at nominal points (slowly varying rotational rate with small pendulum mode). This linear controller implemented in this paper takes advantage of this observation. The restricting condition is that the system is only controllable around the nominal states.

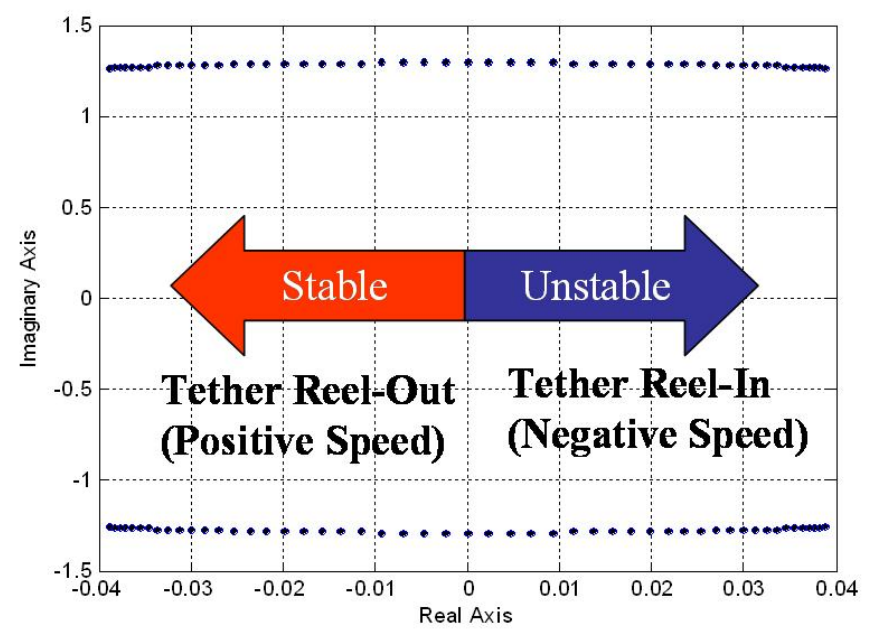

Figure 4. poles of $\phi$ with $-0.5 \mathrm{~m} / \mathrm{s}<i<0.5 \mathrm{~m} / \mathrm{s}, l=1 \mathrm{~m}, \omega=0.3 \mathrm{rad} / \mathrm{s}$

\section{E. Effect of Varying Tether Length}

In the previous sections, the length of the tether is assumed to be invariant $(i=0)$. The dynamics of varying tether length with a constant motor reel-in/out speed $(i=$ constant $)$, is investigated in this section.

Using Eq. (7), we modify the linearized equation (11) as the following:

$$
\left(\begin{array}{c}
\ddot{\theta} \\
\ddot{\phi}
\end{array}\right)+\left[\begin{array}{cc}
2 \frac{i}{l} & 0 \\
-2 \frac{i}{l} & 0
\end{array}\right]\left(\begin{array}{c}
\dot{\theta} \\
\dot{\phi}
\end{array}\right)+\left[\begin{array}{cc}
0 & -\frac{r \omega^{2}\left(I_{G}+m r(r+l)\right)}{l I_{G}} \\
0 & \frac{r \omega^{2}\left(I_{G}+m(r+l)^{2}\right)}{l I_{G}}
\end{array}\right]\left(\begin{array}{c}
\theta \\
\phi
\end{array}\right)=\left[\begin{array}{cc}
\frac{1}{m l} & -\frac{r}{I_{G} l} \\
-\frac{1}{m l} & \frac{r+l}{I_{G} l}
\end{array}\right]\left(\begin{array}{c}
F \\
u
\end{array}\right)
$$

Similarly, the LTI equation (14) is modified into:

$$
\frac{d}{d t}\left(\begin{array}{c}
\theta \\
\phi \\
\dot{\theta} \\
\dot{\phi}
\end{array}\right)=\left[\begin{array}{cccc}
0 & 0 & 1 & 0 \\
0 & 0 & 0 & 1 \\
0 & \frac{r \omega^{2}\left(I_{G}+m r(r+l)\right)}{l I_{G}} & -2 \frac{i}{l} & 0 \\
0 & -\frac{r \omega^{2}\left(I_{G}+m(r+l)^{2}\right)}{l I_{G}} & 2 \frac{i}{l} & 0
\end{array}\right]\left(\begin{array}{c}
\theta \\
\phi \\
\dot{\theta} \\
\dot{\phi}
\end{array}\right)+\left[\begin{array}{cc}
0 & 0 \\
0 & 0 \\
\frac{1}{m l} & -\frac{r}{I_{G} l} \\
-\frac{1}{m l} & \frac{r+l}{I_{G} l}
\end{array}\right]\left(\begin{array}{c}
F \\
u
\end{array}\right)
$$

The nonlinear equations can be easily modified in the same fashion. 
Instead of showing an analytic solutions of the eigenvalues of Eq. (18), a real-imaginary axes plot is presented as shown in figure 4. It is noted that the system is stable when the motor reels out. In other words, a positive reel-out speed resulted in damping of both $\dot{\theta}$ and pendulum motion of $\phi$. However, we will see UNSTABLE motion of states for a negative reel-in speed. In a nutshell, we need a stabilizing controller for the system with decreasing tether length.

\section{Multiple Vehicle Dynamics and Decentralization}

\section{A. Contraction Theory}

\section{Brief Review of Contraction Theory}

Contraction theory developed by Slotine ${ }^{20-22}$ is a simple but unified method to analyze the nonlinear stability of distributed computation and control. Motivated by the biological central nervous system, contraction theory provides a systematic method to build arbitrarily complex systems (tethered formation array) out of simpler elements (a single tethered spacecraft). Combination methods like parallel combination and synchronizing coupling based upon partial contraction theory are explored to simplify the nonlinear dynamics of multiple tethered spacecraft. A brief review of the results from $\operatorname{Ref}^{20-22}$ is presented in this section.

A nonlinear system, possibly a time varying non-autonomous system is formulated as:

$$
\dot{\mathbf{x}}=\mathbf{f}(\mathbf{x}, \mathbf{u}(\mathbf{x}, t), t)
$$

Theorem 1 For the system in Eq. (19), if there exists a uniformly positive definite metric

$$
\mathbf{M}(\mathbf{x}, t)=\Theta(\mathbf{x}, t)^{T} \Theta(\mathbf{x}, t)
$$

where $\Theta$ is some smooth coordinate transformation of the virtual displacement, $\delta \mathbf{z}=\Theta \delta \mathbf{x}$

such that the associated generalized Jacobian,

$$
\mathbf{F}=\left(\dot{\Theta}+\Theta \frac{\partial \mathbf{f}}{\partial \mathbf{x}}\right) \Theta^{-1}
$$

is uniformly negative definite, all system trajectories then converge exponentially to a single trajectory regardless of the initial conditions, with convergence rate of the largest eigenvalues of the symmetric part of $\mathbf{F}$. Then, the system is said to be contracting. Note that for time invariant systems of the form, $\mathbf{x}=\mathbf{f}(\mathbf{x}, u(\mathbf{x}))$, the trajectory converges to an equilibrium point exponentially. This indicates that stability of nonlinear systems can be analyzed more simply by checking the negative definiteness of a proper matrix, rather than finding some implicit motion integral as in Lyapunov theory. Note that contraction theory is a generalization of the classical Kravsovskii theorem. ${ }^{19}$

\section{Contraction of Synchronized System}

The following theorems are used to derive stability of the coupled dynamical systems.

Theorem 2: Parallel Combination ${ }^{21,22}$ Consider two systems of the same dimension, contraction in the same metric,

$$
\dot{\mathbf{x}}=\mathbf{f}_{i}(\mathbf{x}, \mathbf{u}(\mathbf{x}, t), t) i=1,2
$$

Assume further that the metric depends only on the state $\mathbf{x}$ and not explicitly on time. Then, any uniformly positive superposition (where $\exists \alpha>0, \forall t \geq 0, \exists i, \alpha_{i}(t) \geq \alpha$ )

$$
\dot{\mathbf{x}}=\alpha_{1}(t) \mathbf{f}_{1}(\mathbf{x}, \mathbf{u}(\mathbf{x}, t), t)+\alpha_{2}(t) \mathbf{f}_{2}(\mathbf{x}, \mathbf{u}(\mathbf{x}, t), t)
$$

is contracting in the same metric. By recursion, this property of parallel combination can be extended to any number of systems.

Theorem 3: Synchronization ${ }^{22}$ Consider two coupled systems. If the dynamics equations verify

$$
\dot{\mathbf{x}}_{1}-\mathbf{f}\left(\mathbf{x}_{1}, t\right)=\dot{\mathbf{x}}_{2}-\mathbf{f}\left(\mathbf{x}_{2}, t\right)
$$

9 of 26

American Institute of Aeronautics and Astronautics 
where the function $\mathbf{f}(\mathbf{x}, t)$ is contracting, then $\mathbf{x}_{1}$ and $\mathbf{x}_{2}$ will converge to each other exponentially, regardless of the initial conditions.

The theorem is derived by Partial Contraction theory. The proof is given in Ref. ${ }^{22}$

\section{B. Two Spacecraft Dynamics}

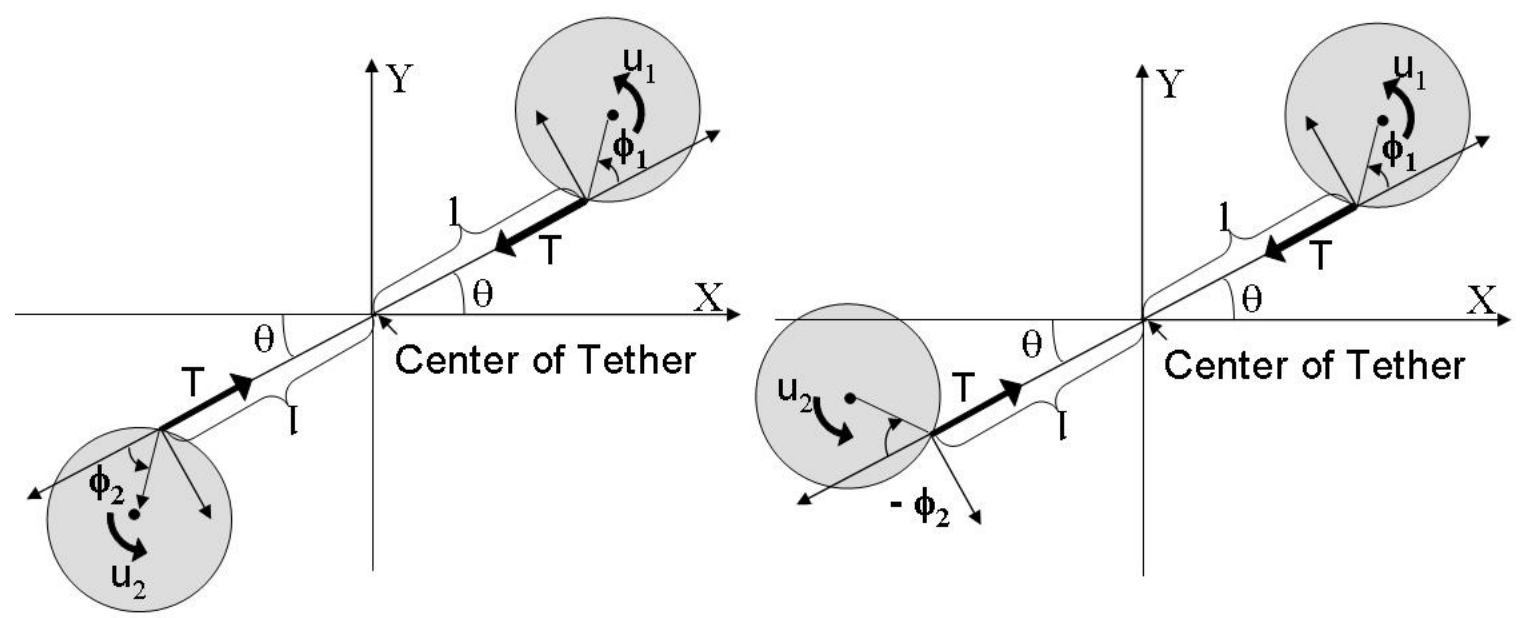

Figure 5. Free-body diagram of tethered two SPHERES. Synchronized $\phi$ (Left), Anti-Synchronized (Right)

The dynamics of two tethered satellites shown in figure 5 is derived using Lagrangian equations. As shown in the figure, the absolute position of the origin of the X-Y axes might freely translate on the 2-D plane but we will focus on the relative dynamics. Note that the positive direction of both $\phi$ angles is always clockwise. The assumption of all identical spacecraft in size, mass and inertia property with torque controls only, allows us to develop the following equation of the motion:

$$
\left[M_{2}\left(\phi_{1}, \phi_{2}\right)\right]\left(\begin{array}{c}
\ddot{\theta} \\
\ddot{\phi_{1}} \\
\ddot{\phi_{2}}
\end{array}\right)+\left[C_{2}\left(\phi_{1}, \phi_{2}, \dot{\theta}, \dot{\phi_{1}}, \dot{\phi_{2}}\right)\right]=\left(\begin{array}{c}
u_{1}+u_{2} \\
u_{1} \\
u_{2}
\end{array}\right)
$$

where $\left[M_{2}\right]=\left[\begin{array}{ccc}2 I_{G}+2 m r^{2}+2 m l^{2}+2 m r l \cos \phi_{1}+2 m r l \cos \phi_{2} & I_{G}+m r^{2}+m r l \cos \phi_{1} & I_{G}+m r^{2}+m r l \cos \phi_{2} \\ I_{G}+m r^{2}+m r l \cos \phi_{1} & I_{G}+m r^{2} & 0 \\ I_{G}+m r^{2}+m r l \cos \phi_{2} & 0 & I_{G}+m r^{2}\end{array}\right]$ and

$$
\left[C_{2}\left(\phi_{1}, \phi_{2}, \dot{\theta}, \dot{\phi_{1}}, \dot{\phi_{2}}\right)\right]=\left[\begin{array}{c}
-2 m r l \dot{\theta}\left(\sin \phi_{1} \dot{\phi}_{1}+\sin \phi_{2} \dot{\phi}_{2}\right)-m r l \sin \phi_{1} \dot{\phi}_{1}^{2}-m r l \sin \phi_{2}{\dot{\phi_{2}}}^{2} \\
m r l \sin \phi_{1} \dot{\theta}^{2} \\
m r l \sin \phi_{2} \dot{\theta}^{2}
\end{array}\right]
$$

The tether length is now $2 l$. The array angular rate $\dot{\theta}$ is assumed to be the same for both satellites. This is especially true when the tether is in tension. Furthermore, the mass and inertia properties are assumed to be roughly the same. It can be inferred by inspecting Eq. (25) that the first line of the equation is the only coupled equation, which is the sum of two independent single tethered system of the form in Eq. (8):

$$
\begin{aligned}
& {\left[M_{1}\left(\phi_{1}\right)\right]\left(\begin{array}{c}
\ddot{\theta} \\
\ddot{\phi_{1}}
\end{array}\right)+\left[C_{1}\left(\phi_{1}, \dot{\theta}, \dot{\phi}_{1}\right)\right]=\left(\begin{array}{c}
u_{1} \\
u_{1}
\end{array}\right)} \\
& {\left[M_{1}\left(\phi_{2}\right)\right]\left(\begin{array}{c}
\ddot{\theta} \\
\ddot{\phi_{2}}
\end{array}\right)+\left[C_{1}\left(\phi_{2}, \dot{\theta}, \dot{\phi}_{2}\right)\right]=\left(\begin{array}{c}
u_{2} \\
u_{2}
\end{array}\right)}
\end{aligned}
$$




$$
\begin{gathered}
\text { where }\left[M_{1}(\phi)\right]=\left[\begin{array}{cc}
I_{G}+m r^{2}+m l^{2}+2 m r l \cos \phi & I_{G}+m r^{2}+m r l \cos \phi \\
I_{G}+m r^{2}+m r l \cos \phi & I_{G}+m r^{2}
\end{array}\right] \text { and } \\
{\left[C_{1}(\phi, \dot{\theta}, \dot{\phi})\right]=\left[\begin{array}{c}
-2 m r l \sin \phi \dot{\theta} \dot{\phi}-m r l \sin \phi \dot{\phi}^{2} \\
m r l \sin \phi \dot{\theta}^{2}
\end{array}\right]}
\end{gathered}
$$

It is easily verified that Eq. (25) gets reduced to Eqs. (26-27) if $\phi_{1}$ and $\phi_{2}$ can somehow be synchronized. In other words, we can decouple the dynamics of the first SPHERES from the other resulting in the same equation as Eq. (8) when the controller $u_{1}$ and $u_{2}$ make $\phi_{1}$ and $\phi_{2}$ converge towards each other exponentially fast. This important finding leads to the following theorem.

Theorem 4: Decentralized Control Law suppose that for two tethered spacecraft, some control function $u$ is decentralized in a sense that it does not require state information from the other satellite. It may be written as:

$$
u_{1}=u\left(\phi_{1}, \dot{\theta}, \dot{\phi}_{1}\right) \text { and } u_{2}=u\left(\phi_{2}, \dot{\theta}, \dot{\phi}_{2}\right) \text { where } \mathrm{u} \text { is the same control function }
$$

If $u_{1}$ and $u_{2}$, strictly stabilizing two single tethered systems in Eq. (26) and (27) respectively, also make the combined system in Eq. (25) strictly stable, then a decentralized control law, $u_{i}=u\left(\phi_{i}, \dot{\theta}, \dot{\phi}_{i}\right), i=1,2$ can be designed from a single tethered system in Eqs. (26-27) in lieu of the two body dynamics in Eq. (25). In addition, this control law $u$ makes $\phi_{1}$ and $\phi_{2}$ converge to each other.

This will significantly simplify both control algorithm and satellite hardware with less complication in terms of dimensionality and no communication burden. Basically, the fixed center of the rotation for a single tethered system will be replaced with the center of the tether of the two SPHERES (see figure 5). The subsequent sections will prove the existence of such controller as in Theorem 4 in two ways: by normalizing transformation (Section C) and by contraction theory (Section D).

\section{Stability of Decentralized Control Law}

Two spacecraft equation (25) can also be linearized about $\dot{\theta}=\omega$, and $\dot{\phi}, \phi=0$ :

$\left[\begin{array}{ccc}2 I_{G}+2 m(r+l)^{2} & I_{G}+m r(r+l) & I_{G}+m r(r+l) \\ I_{G}+m r^{2}+m r l & I_{G}+m r^{2} & 0 \\ I_{G}+m r^{2}+m r l & 0 & I_{G}+m r^{2}\end{array}\right]\left(\begin{array}{c}\ddot{\theta} \\ \ddot{\phi_{1}} \\ \ddot{\phi_{2}}\end{array}\right)+\left[\begin{array}{ccc}0 & 0 & 0 \\ 0 & m r l \omega^{2} & 0 \\ 0 & 0 & m r l \omega^{2}\end{array}\right]\left(\begin{array}{c}\theta \\ \phi_{1} \\ \phi_{2}\end{array}\right)=\left(\begin{array}{c}u_{1}+u_{2} \\ u_{1} \\ u_{2}\end{array}\right)$

The natural frequencies of this LTI system are computed as

$$
\left(\begin{array}{c}
\omega_{\theta} \\
\omega_{\phi} \\
\omega_{o}
\end{array}\right)=\left(\begin{array}{c}
\frac{0}{\frac{r\left(I_{G}+m(r+l)^{2}\right)}{l I_{G}}} \omega \\
\sqrt{\frac{m r l}{I_{G}+m r^{2}}} \omega
\end{array}\right)
$$

where $\omega_{\phi}$ is the same pendulum mode frequency from the single spacecraft case as Eq. (13), and $\omega_{\theta}=0$ is the same rigid body mode of $\theta$ and $\dot{\theta}$ whereas $\omega_{o}$ is the natural frequency of anti-synchronization mode depicted in figure 5 . It can be shown that $\omega_{o}$ is always smaller than $\omega_{\phi}$.

We can diagonalize the system in Eq. (29) using normalized eigenvectors. Then, with a linear controller of the form:

$$
u_{i}=K_{1} \phi_{i}+K_{2} \dot{\theta}+K_{3} \dot{\phi}_{i} i=1,2
$$

Eq. (29) becomes 


$$
[V]^{T}[M][V]\left(\begin{array}{c}
\ddot{z}_{1} \\
\ddot{z}_{2} \\
\ddot{z}_{3}
\end{array}\right)+[V]^{T}[K][V]=[V]^{T}\left[\begin{array}{ccc}
0 & K_{1} & K_{1} \\
0 & K_{1} & 0 \\
0 & 0 & K_{1}
\end{array}\right][V]\left(\begin{array}{c}
z_{1} \\
z_{2} \\
z_{3}
\end{array}\right)+[V]^{T}\left[\begin{array}{ccc}
2 K_{2} & K_{3} & K_{3} \\
K_{2} & K_{3} & 0 \\
K_{2} & 0 & K_{3}
\end{array}\right][V]\left(\begin{array}{c}
\dot{z}_{1} \\
\dot{z}_{2} \\
\dot{z}_{3}
\end{array}\right)
$$

where constant matrices $[M]$ and $[K]$ are from Eq. (29), and

$[V]$ denotes a matrix of normalized eigenvectors sorted in the same order as Eq. (30):

$$
[V]=\left[\begin{array}{ccc}
\frac{1}{\sqrt{2 I_{G}+2 m(r+l)^{2}}} & -\frac{I_{G}+m(r+l)}{\sqrt{2 I_{G} l^{2} m} \sqrt{I_{G}+m(r+l)^{2}}} & 0 \\
0 & \frac{\sqrt{I_{G}+m(r+l)^{2}}}{\sqrt{2 I_{G} l^{2} m}} & -\frac{1}{\sqrt{2 I_{G}+2 m r^{2}}} \\
0 & \frac{\sqrt{I_{G}+m(r+l)^{2}}}{\sqrt{2 I_{G} l^{2} m}} & \frac{1}{\sqrt{2 I_{G}+2 m r^{2}}}
\end{array}\right]
$$

Since $[V]^{T}[M][V]=I_{3 \times 3}$ and $[V]^{T}[K][V]=\operatorname{diag}\left(0, \omega_{\phi}^{2}, \omega_{o}^{2}\right)$, Eq. (32) becomes:

$$
\begin{gathered}
\ddot{z}_{1}=q_{1} \dot{z}_{1}+q_{2} \dot{z}_{2}+p_{1} z_{2} \\
\ddot{z}_{2}+\omega_{\phi}^{2} z_{2}=q_{3} \dot{z}_{1}+q_{4} \dot{z}_{2}+p_{2} z_{2} \\
\ddot{z}_{3}-\frac{K_{3}}{I_{G}+m r^{2}} \dot{z}_{3}+\left(\omega_{o}^{2}-\frac{K_{1}}{I_{G}+m r^{2}}\right) z_{3}=0
\end{gathered}
$$

where $q_{1}=\frac{K_{2}}{I_{G}+m(r+l)^{2}}, q_{2}=\frac{I_{G}\left(-K_{2}+K_{3}\right)+m(r+l)\left(-r K_{2}+K_{3}(r+l)\right)}{\sqrt{I_{G} l^{2} m}\left(I_{G}+m(r+l)^{2}\right)}, q_{3}=\frac{m l(r+l)}{\sqrt{I_{G} l^{2} m}} q_{1}, q_{4}=(r+l) \sqrt{\frac{m}{I_{G}}} q_{2}$, $p_{1}=\frac{K_{1}}{\sqrt{I_{G} l^{2} m}}$, and $p_{2}=\frac{K_{1}(r+l)}{I_{G} l}$

By inspecting the eigenvectors in Eq. (33), it is clear that $z_{1}$ represents $\theta$ and $z_{2}$ is the dynamics of the synchronized pendulum mode $\left(\phi_{1}+\phi_{2}\right)$ in figure 5 with the same natural frequency $\omega_{\phi}$ as the single tethered case. The additional mode is the pendulum mode of anti-synchrony, which is $z_{3} \propto \phi_{1}-\phi_{2}$.

Likewise, if we diagonalize the linearized single spacecraft system in Eq.(10), the same normalized equations of motions for $z_{1}$ and $z_{2}$ in Eq. (34) are produced. Thus, the stabilizing controller $u_{i}$ will stabilize $\theta$ and the synchronized pendulum mode $z_{2}$. Now proving Theorem 4 comes down to finding the conditions in which anti-synchronized mode $z_{3}$ is stabilized.

Using contraction theory in section A, we can find the stability condition of $K_{1}$ and $K_{3}$ using the following transformation for $x=z_{3}$ :

$$
\left(\begin{array}{l}
\dot{x} \\
\dot{y}
\end{array}\right)=\left[\begin{array}{cc}
a & \omega_{o} \\
-\omega_{o} & b
\end{array}\right]\left(\begin{array}{l}
x \\
y
\end{array}\right)
$$

where $a+b=\frac{K_{3}}{I_{G}+m r^{2}}$ and $a b=-\frac{K_{1}}{I_{G}+m r^{2}}$

The generalized Jacobian from Eq. (21), $\mathbf{F}=\left[\begin{array}{cc}a & \omega_{0} \\ -\omega_{0} & b\end{array}\right]$ is negative definite if and only if $a<0$, $b<0$, which in turn corresponds to $K_{3}<0$ and $K_{1}<0$.

Similarly, we can find the stability condition of $z_{1}$ and $z_{2}$, which is characterized like the following,

Theorem 5 The following conditions should be satisfied to stabilize a single tethered system with a decentralized linear control law in Eq. (31),

$$
r K_{2}>(r+l) K_{3}, K_{1}<0, K_{2}<0
$$

It is straightforward to show that any controller satisfying Theorem 5 will stabilize the mode $z_{3}$, thereby stabilizing the coupled two-body system in Eq. (29). This proves Theorem 4.

This result is graphically illustrated in figure 6 . Since $(r+l) / r=(0.125 m+1 m) / 0.125 m=9$, the system is strictly stable until $K_{2}$ reaches $9 K_{3}=1.8$ as shown in the left figure. And it is shown that the anti-synchronizing mode $\left(z_{3}\right)$ is independent of $K_{2}$. Moreover, the poles of the controlled single tethered 

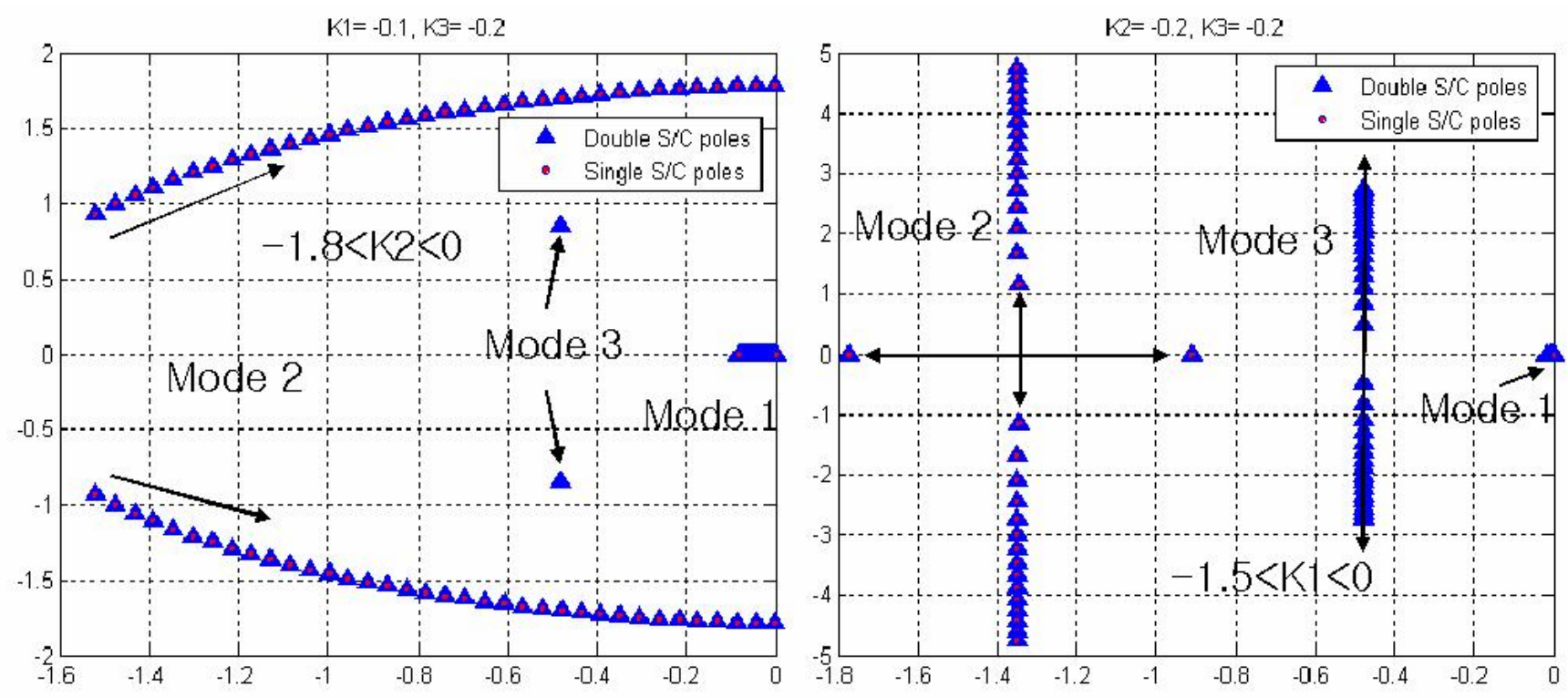

Figure 6. Poles of two tethered spacecraft and single spacecraft with linear control

system are the poles of $z_{1}$ and $z_{2}$ in Eq. (34) as shown in the figure.

Theorem 6 When the tether motors reel in or out, $i$ is a nonzero term. It can be shown that $i$ does not affect the anti-synchronizing mode $z_{3}$, therefore the controller stabilizing a single tethered system with the tether length varying at a constant speed (section III-E) can be used. i.e. Theorem 4 also holds for nonzero tether speed, $i$ as long as $i$ is the same for both satellites.

\section{Stability by Contraction Theory}

Stability of anti-synchronizing pendulum mode $z_{3}$ in Eq. (34) can be verified more easily using contraction theory, specifically Theorem 2 and $\mathbf{3}$. From 2nd and 3rd row of Eq. (29),

$$
\begin{aligned}
& \left(I_{G}+m r^{2}+m r l\right) \ddot{\theta}+\left(I_{G}+m r^{2}\right) \ddot{\phi}_{1}+m r l \omega^{2} \phi_{1}=K_{1} \phi_{1}+K_{3} \dot{\phi}_{1}+K_{2} \dot{\theta} \\
& \left(I_{G}+m r^{2}+m r l\right) \ddot{\theta}+\left(I_{G}+m r^{2}\right) \ddot{\phi}_{2}+m r l \omega^{2} \phi_{2}=K_{1} \phi_{2}+K_{3} \dot{\phi}_{2}+K_{2} \dot{\theta}
\end{aligned}
$$

Subtracting the 1st line from the 2nd in Eq. (37) results in:

$$
\left(I_{G}+m r^{2}\right) \ddot{\phi}_{1}+m r l \omega^{2} \phi_{1}-K_{1} \phi_{1}-K_{3} \dot{\phi}_{1}=\left(I_{G}+m r^{2}\right) \ddot{\phi}_{2}+m r l \omega^{2} \phi_{2}-K_{1} \phi_{2}-K_{3} \dot{\phi}_{2}
$$

From Theorem 3, if the $z_{3}$ dynamics in Eq. (34) is exponentially stable, then $\phi_{1}$ converges to $\phi_{2}$ exponentially fast. Then, the stability of the original systems depends on the stability of the single tethered system. Futhermore, $\left(I_{G}+m r^{2}+m r l\right) \ddot{\theta}$ can be regarded as an auxiliary input of the partially contracting dynamics of $z_{3}$. Therefore, a stable closed-loop single tethered dynamics stabilizes $z_{3}$ dynamics.

More rigorously, Theorem 4 can be proven by parallel combination of contracting systems (Theorem 2).

Multiplying Eq. (29) with the inverse matrix of $M_{2}$ results in:

$$
\left(\begin{array}{c}
\ddot{\theta} \\
\ddot{\phi_{1}} \\
\ddot{\phi_{2}}
\end{array}\right)=\left[\begin{array}{cc}
c & c \\
-k_{1} & -k_{2} \\
-k_{2} & -k_{1}
\end{array}\right]\left(\begin{array}{l}
\phi_{1} \\
\phi_{2}
\end{array}\right)+\left[\begin{array}{cc}
-b & -b \\
b_{1} & b_{2} \\
b_{2} & b_{1}
\end{array}\right]\left(\begin{array}{l}
u_{1} \\
u_{2}
\end{array}\right)
$$


where

$$
\begin{array}{r}
c=\frac{r\left(I_{G}+m r(l+r)\right) \omega^{2}}{2 I_{G} l} \\
k_{1}=\frac{r\left(I_{G}^{2}+2 m\left(l^{2}+r l+r^{2}\right) I_{G}+m^{2} r^{2}(l+r)^{2}\right) \omega^{2}}{2 I_{G} l\left(m r^{2}+I_{G}\right)} \\
k_{2}=\frac{r\left(I_{G}+m r(l+r)\right)^{2} \omega^{2}}{2 I_{G} l\left(m r^{2}+I_{G}\right)} \\
b=\frac{r}{2 I_{G} l}, b_{1}=\frac{1}{2}\left(\frac{l+r}{I_{G} l}+\frac{1}{m r^{2}+I_{G}}\right), \text { and } b_{2}=\frac{r\left(I_{G}+m r(l+r)\right)}{2 I_{G} l\left(m r^{2}+I_{G}\right)}
\end{array}
$$

It can be easily verified that $k_{1}+k_{2}=\omega_{\phi}^{2}$ and $k_{1}-k_{2}=\omega_{o}^{2}$.

The LTI equation of a single tethered system in Eq. (14) can be written as

$$
\left(\begin{array}{c}
\ddot{\theta} \\
\ddot{\phi}_{i}
\end{array}\right)=\left[\begin{array}{c}
2 c \\
-\omega_{\phi}^{2}
\end{array}\right] \phi_{i}+\left[\begin{array}{c}
-2 b \\
\frac{r+l}{I_{G} l}
\end{array}\right] u_{i}=\left(\begin{array}{c}
f_{\theta, i} \\
f_{\phi, i}
\end{array}\right), i=1,2
$$

Using the equation above, Eq. (39) can be re-written as:

$$
\left(\begin{array}{c}
\ddot{\theta} \\
\ddot{\phi}_{1} \\
\ddot{\phi}_{2}
\end{array}\right)=\left(\left[\begin{array}{cc}
\frac{1}{2}\left(f_{\theta, 1}+f_{\theta, 2}\right) \\
k_{1} & k_{2} \\
k_{2} & k_{1}
\end{array}\right]\left(\begin{array}{c}
f_{\phi, 1} / \omega_{\phi}^{2} \\
f_{\phi, 2} / \omega_{\phi}^{2}
\end{array}\right)\right)
$$

Since $k_{1}-k_{2}>0$ and $k_{1}+k_{2}>0$ for nonzero array angular speed $\omega$, the matrix $\left[\begin{array}{ll}k_{1} & k_{2} \\ k_{2} & k_{1}\end{array}\right]$ is always positive definite. In conclusion, this is truly uniformly positive superposition of two contracting systems as in Theorem 2. Accordingly, a tethered two spacecraft system is contracting as long as it is composed of two single tethered system with contracting $f_{\theta, i}$ and $f_{\phi, i}, i=1,2$. This is another proof of Theorem 4, which is a lot simpler than the proof in the previous section.

\section{E. Three Spacecraft Dynamics}

Two configurations for three tethered spacecraft are being considered for the SPECS mission as shown in figure 7. The performance of two configurations are yet to be compared in the near future. Motivated by successful decoupling for the two spacecraft case, three imaginary pseudo-tethers connecting each satellite to the Center of Mass (CM) of the array are assumed to be present replacing the three actual tether lines (see the dashed lines in figure 7). When the tethers are taut and straight in a rotating array, a small $\phi$ (angle of pendulum mode) is approximated as a perturbed angle that the SPHERES makes with respect to the corresponding pseudo-tether. Then, the formulation of the dynamics of each SPHERES results in the single tether case Eq. (6) and (8). The length of the pseudo-tether is the actual tether length divided by $\sqrt{3}$. This pseudo-tether technique is yet to be compared with the actual dynamics obtained by Lagrange's equation. However, the controller based on this technique performed well in the experiments at the MSFC floor in December 2004. Similarly, the dynamics of the inline configuration can be decoupled into two independent dynamics of a slightly modified version of the single tethered system and rotational dynamics of the center bus. Nonlinear decentralized controller using tether tension measurements are currently under development for three inline configuration.

\section{Sensor Suite and Estimation}

\section{A. Current Measurement System}

Any control algorithms require that all the states in the dynamics of the previous sections, are strictly available for feedback. The role of the relative estimator is to provide autonomous state estimates (tether length, and all the states including the bearing angle $\phi$ ) independently of adjacent satellites. It is desirable

to maintain the independency to avoid any possible communication delays between satellites. Estimation of 


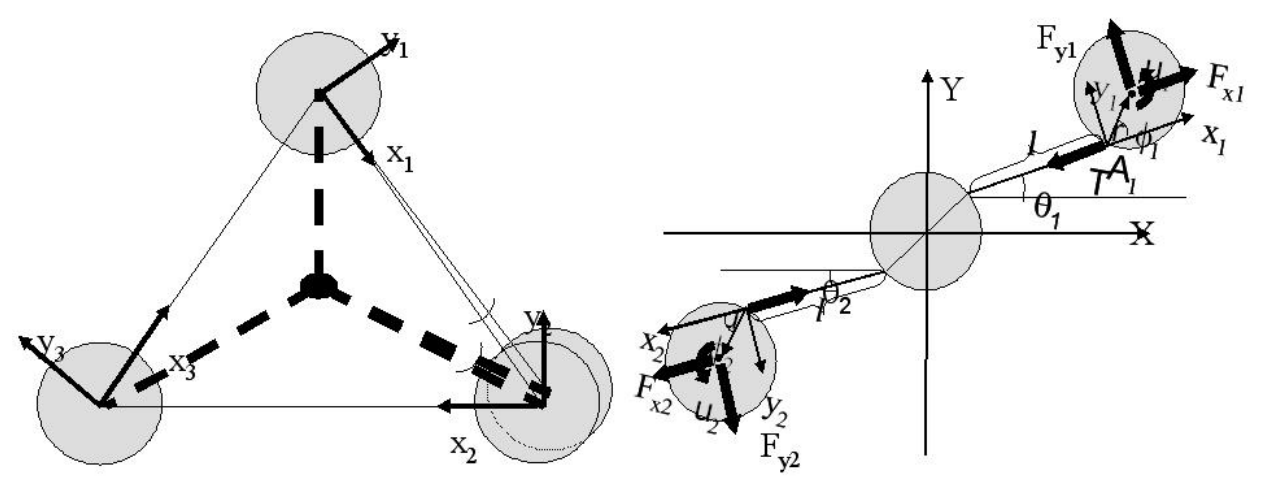

Figure 7. Two possible configurations for three tethered satellites for SPECS

relative attitude is realized by the dynamics in section III, and state estimation of multiple vehicles can be simplified into that of the single tethered spacecraft case as proved in IV. In this section, a description on the relative metrology sensor suite and estimator design to estimate the system states $(\phi, \dot{\theta}, \dot{\phi})$ is elaborated.

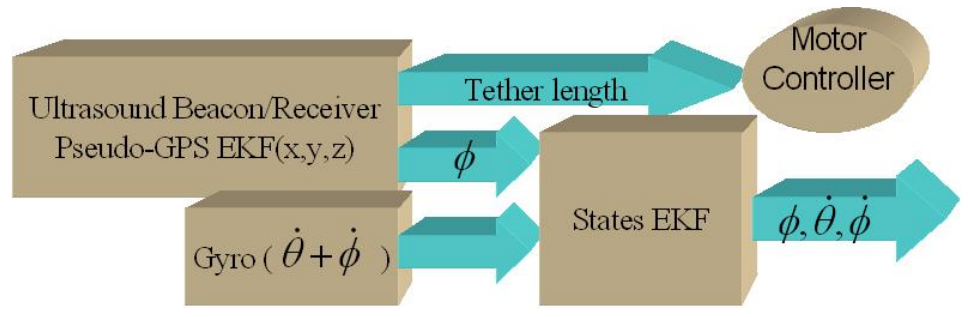

Figure 8. Pendulum angle determination using relative metrology system

Each SPHERES satellite is equipped with a beacon located on its docking face. An additional on-board beacon is also mounted on the expansion port face where the tether mechanism package is attached (See figure 2). The ultrasound system measures $\mathrm{x}, \mathrm{y}, \mathrm{z}$, coordinate values of the beacon attached to the second SPHERES with respect to the local frame of the first SPHERES. Then, the bearing angle $\phi$ and the range $l$ are easily derived from the $\mathrm{x}, \mathrm{y}, \mathrm{z}$ values using the geometry.

This U/S ranging system estimates the $\mathrm{x}, \mathrm{y}, \mathrm{z}$ coordinates of the incoming beacon in the body frame of the SPHERES as depicted in figure 9. The measurement process is similar to using the time gap between seeing the lightening and hearing the thunder clap to calculate how far you are away from a thunderstorm. The method by which the on-board beacons are used to compute relative position and attitude is as following. When the leader SPHERES requests a next state update, it emits an infrared ping which is received by all the surrounding SPHERES and the external on-board beacons. This is a time-synchronization process. All the SPHERES then start to listen for an U/S ping through their $24 \mathrm{U} / \mathrm{S}$ receivers located all around them. The beacons emit an US ping one after the other (20 milliseconds apart) to avoid the interference. The pings are then received by each SPHERES and an array of time-of-flight data is sent to the computer. Since the range measurement is nonlinear, an extended Kalman filter is employed to estimate the range in terms of $\mathrm{x}, \mathrm{y}, \mathrm{z}$, coordinate values.

Once the pseudo-GPS system estimates $\mathrm{x}, \mathrm{y}, \mathrm{z}$ coordinates of the target beacon, the tether length $l$ and the bearing pendulum mode angle $\phi$ are computed from the geometry shown in figure 9 :

$$
\begin{gathered}
l=\sqrt{(x-r)^{2}+y^{2}+z^{2}} \\
\phi=\arcsin (y / l)
\end{gathered}
$$

The tether length is used in realtime for the tether-motor control whereas the direct $\phi$ measurement is used as complementary filtering with the high-frequency gyro measurement (see figure 8). 


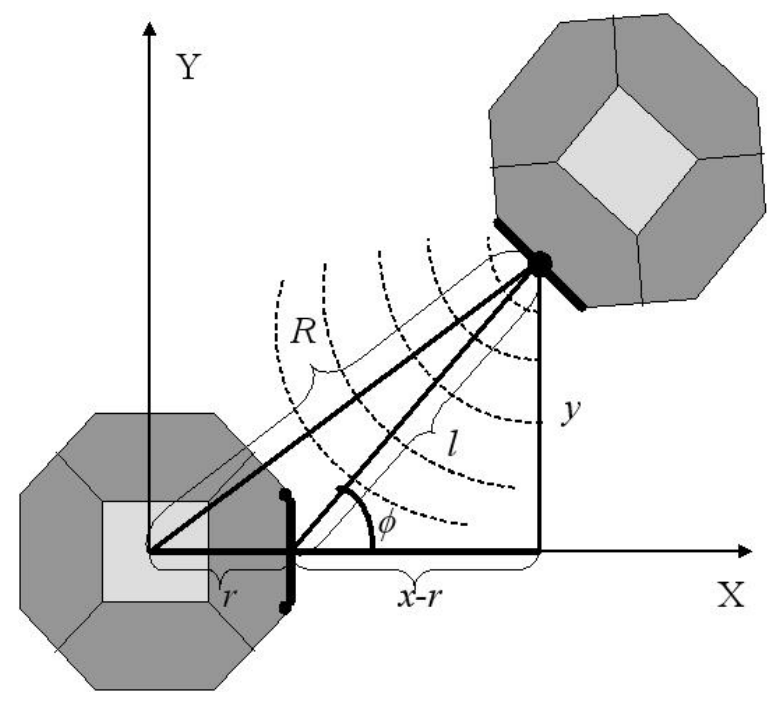

Figure 9. Pendulum angle determination using relative metrology system

The complementary extended Kalman filtering makes use of the nonlinear equation developed in section III in order to estimate $\phi, \dot{\theta}$, and $\dot{\phi}$. It can be formulated as follows:

\section{Propagation}

$$
\begin{aligned}
& \frac{d}{d t}\left(\begin{array}{c}
\hat{\phi} \\
\hat{\dot{\theta}} \\
\hat{\dot{\phi}}
\end{array}\right)= \\
& -\frac{1}{l\left(I_{G}+m r^{2}(\sin \hat{\phi})^{2}\right)}\left[\begin{array}{c}
-l\left(I_{G}+m r^{2}(\sin \hat{\phi})^{2}\right) \hat{\dot{\phi}} \\
r \sin \hat{\phi}\left[\left(I_{G}+m r^{2}\right)(\hat{\dot{\theta}}+\hat{\dot{\phi}})^{2}+m r l \hat{\dot{\theta}}^{2} \cos \hat{\phi}+m l^{2} \hat{\dot{\theta}} \dot{\hat{\theta}}^{2} \cos \hat{\phi}\right] \\
-r \sin \hat{\phi}\left[\left(I_{G}+m r^{2}(\hat{\dot{\theta}}+\dot{\hat{\phi}})^{2} \cos \hat{\phi}\right]\right.
\end{array}\right] \\
& +\frac{1}{l\left(I_{G}+m r^{2}(\sin \hat{\phi})^{2}\right)}\left(\begin{array}{c}
0 \\
-r \cos \hat{\phi} \\
l+r \cos \hat{\phi}
\end{array}\right) u \\
& \dot{P}=A(\hat{x}) P+P A(\hat{x})^{\prime}+Q
\end{aligned}
$$

$P$ is the error covariance matrix and $Q$ is the covariance matrix of the process noise.

\section{Measurement Update}

$$
\begin{gathered}
\left.x_{k} \hat{(+}\right)=x_{k} \hat{(-)}+L_{k}\left(y_{k}-C x_{k}(-)\right) \\
L_{k}=P_{k}(-) C^{\prime}\left[C P_{k}(-) C^{\prime}+R_{k}\right]^{-1} ; \\
P_{k}(+)=\left(I-L_{k} C\right) P_{k}(-)\left(I-L_{k} C\right)^{\prime}+L R_{k} L^{\prime}
\end{gathered}
$$

where the measurement vector $y_{k}=\left[\begin{array}{c}\text { gyro measurement } \\ \phi \text { from U/S beacon }\end{array}\right]$ and $C=\left[\begin{array}{lll}0 & 1 & 1 \\ 1 & 0 & 0\end{array}\right] . L_{k}$ denotes the optimal Kalman gain and $R_{k}$ is the discrete covariance matrix of the sensor noise. 
It is noted that the Joseph form of the covariance measurement update is used to maintain the symmetric property.

\section{B. New Bearing Angle Measurement Mechanism}

The current U/S $\phi$ angle measurement system theoretically has a three millimeter resolution in range. A real-world test gives a one centimeter resolution. This will results in mere a few degree resolution in bearing angle for $20 \mathrm{~cm}$ tether length. In addition, undesirable high-spikes are sometimes present affecting the performance of the system. Those high-spikes are suspected to be generated from the environmental noise and the reflectivity of the surface.

The usage of ultrasound wave also gives rise to fatal limitations as applied to the tethered system: It does not work well in close proximity due to the cone angle $(+/-30$ degrees) of U/S beacon. It turned out from the experiment that measurements within $15 \mathrm{~cm}$ are very poor. And this minimum working distance dramatically decreases with large bearing angles. Moreover, it does not correctly measure large angles (e.g. more than 90 degrees) since the U/S system always needs to maintain clear line-of-sight between the receiver face and the beacon face. This is very problematic since we are trying to control the formation with reaction wheels only (no thrusters). This is inherently underactuated system actuating the whole system only using the reaction wheels (see Eq. 16), and may involve large pendulum mode angle $(\phi)$ at transient stages. Moreover, this problem is more pronounced for large initial angles with triangular configurations (see figure 2). The face of $\mathrm{U} / \mathrm{S}$ receives make 60 degrees with the beacon face even with zero $\phi$ angles, thereby reducing the reliability of the system.

It also has a limitation in the bandwidth. To avoid confusion, each SPHERES needs to identify each beacon number separated by some time gap $(20 \mathrm{~ms})$. For three SPHERES, we need three relative beacons. We can run this U/S metrology system up to $10 \mathrm{~Hz}$. This is also limited by the computation load imposed on the DSP by complex extended Kalman filter algorithms. Also note neither tether tension nor slackness can be measured by the current system.

New bearing angle measurement system using force-torque (F/T) sensor outputs is under development and proven to resolve all the issues identified in this section. A brief summary of the current design is presented in this paper.

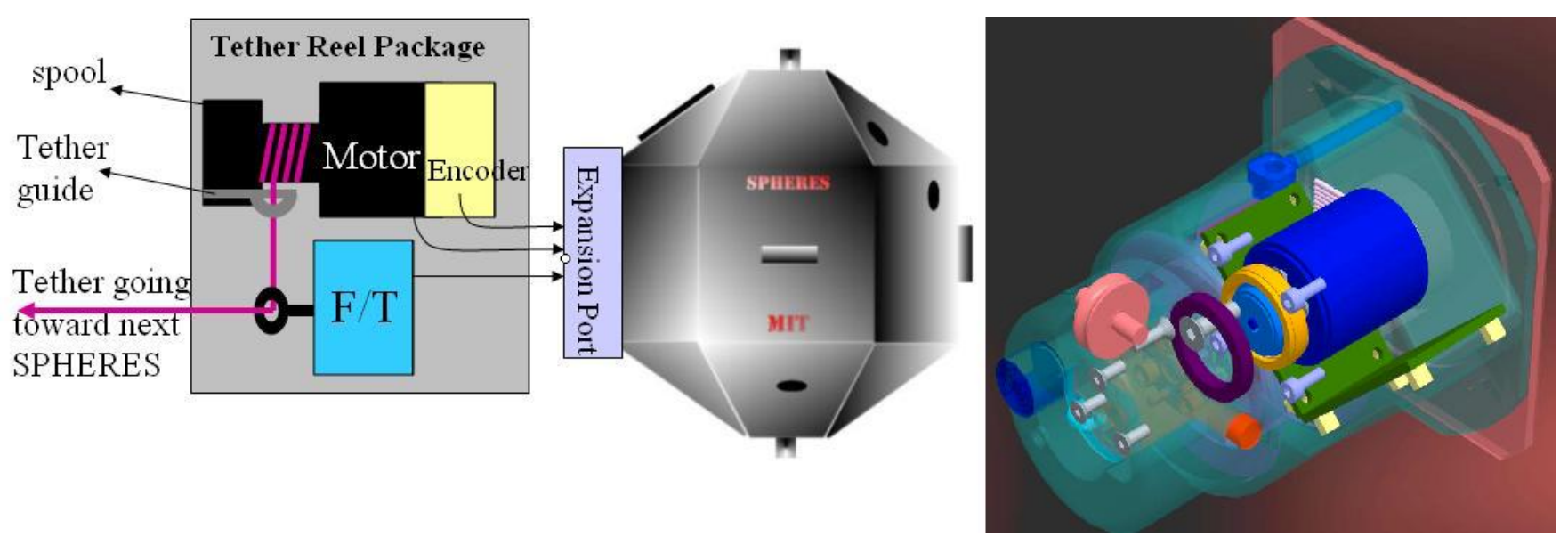

Figure 10. Schematic of tether mechanism with F/T sensor (left) and CAD drawing (right)

As shown in Figure 10, the tether coming from the motor spool will make 90 degrees with the tether end that goes towards the other SPHERES. Forces are exerted on the F/T sensor through a frictionless roller or ring which can pass the incidence forces to the load cell appropriately.

From the force free diagram of this setup (Figure 11), we can determine the tension and bearing angle using the following equations: 


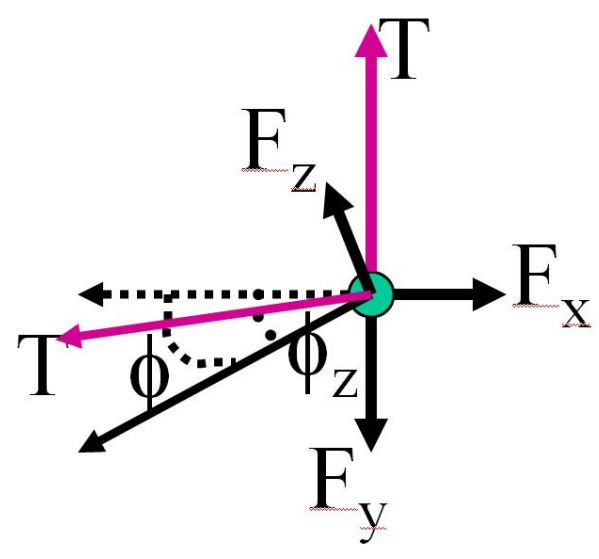

Figure 11. Force free diagram

$$
\begin{array}{r}
\Sigma_{x}: T-F_{y}=T \cos \phi_{z} \sin \phi(\mathrm{x} \text { forces }) \\
\Sigma_{y}: F_{x}=T \cos \phi_{z} \cos \phi(\mathrm{y} \text { forces }) \\
\Sigma_{z}: F_{z}=T \sin \phi_{z}(\mathrm{z} \text { forces })
\end{array}
$$

Then using the trigonometric relationship $\sin \phi^{2}+\cos \phi^{2}=1$ and the previous equations, we can solve for $T, \phi$ (planar pendulum mode angle) and $\phi_{z}$ (vertical angle) as the following:

$$
\begin{aligned}
& T=\frac{F_{x}{ }^{2}+F_{y}{ }^{2}+F_{z}{ }^{2}}{2 F_{y}} \\
& \phi=\tan ^{-1} \frac{F_{x}{ }^{2}+F_{z}{ }^{2}-F_{y}{ }^{2}}{2 F_{x} F_{y}} \\
& \phi_{z}=\sin ^{-1} \frac{2 F_{y} F_{z}}{{F_{x}}^{2}+F_{z}{ }^{2}-F_{y}{ }^{2}}
\end{aligned}
$$

where $\mathrm{T}$ is tension of the tether which remains the same throughout the length for inextensible, massless tether. $F_{x}, F_{y}$ and $F_{z}$ denote force measurements of load cell in the x,y directions respectively.

Raw force-torque outputs from the load cell are transferred thru a serious of filters and signal conditioners to the DSP of the SPHERES. In essence, the analog low pass filter avoids the anti-aliasing of high frequency noise while the digital low pass filter rejects the high-frequency noises or vibrations beyond the frequency region of interest. The sensitivity matrix converts the raw voltage 6 channel outputs to the proper load units (in $\mathrm{N}$ and $\mathrm{Nm}$ ). The off-axis components in the sensitivity matrix indicate the coupling of the strain-gauges.

\section{Linear Control and Experimental Results}

Section VI and VII include experiment results obtained at the NASA Marshall Space Flight Center, December 2004. The measurement system for these experiments is the ultrasound metrology with gyro as illustrated in section V-A. More experiments will be conducted in 2005 with new hardware. Now we can easily construct a linear controller with the single tethered LTI system given by Eq. (14) using torque input $u$ only. We intentionally eliminate the state $\theta$ from Eq. (14) since it is merely a rigid body mode. So all the states are available by the estimator in the previous section. We can design a Linear Quadratic Regulator 


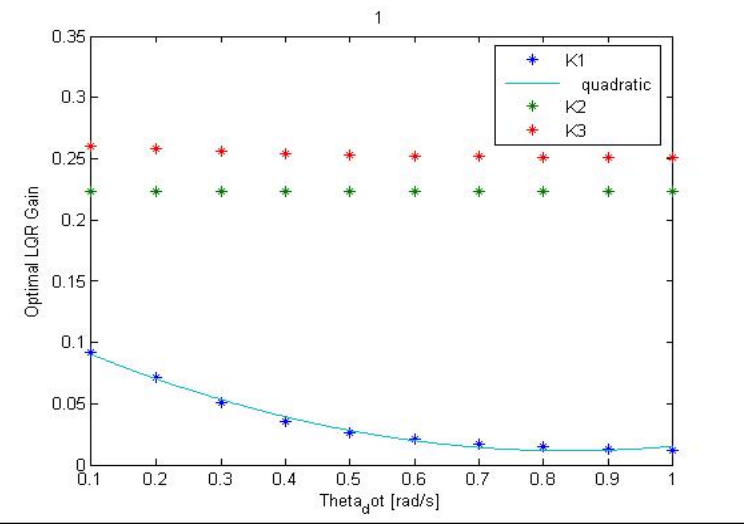

Figure 12. LQR gains for three states scheduled over various $\dot{\theta}$

(LQR) controller, which minimizes the following cost function,

$$
J=\int_{0}^{\infty}\left[x^{T} Q x+u^{T} R u\right] d t
$$

The LQR controller specifically addresses the issue of achieving a balance between good system response and the control effort required. Since the A matrix is a function of $\dot{\theta}$ and the tether length $l$, the optimal LQR gains are calculated over various angular rate and tether length. Since we are going to increase the tether length after achieving a certain angular rate, a gain-scheduled LQR gain is a continuous function of $\dot{\theta}$ over discrete tether length as depicted in figure 12. Some of the curve-fitted functions resulted in a nonlinear quadratic function of $\dot{\theta}$.
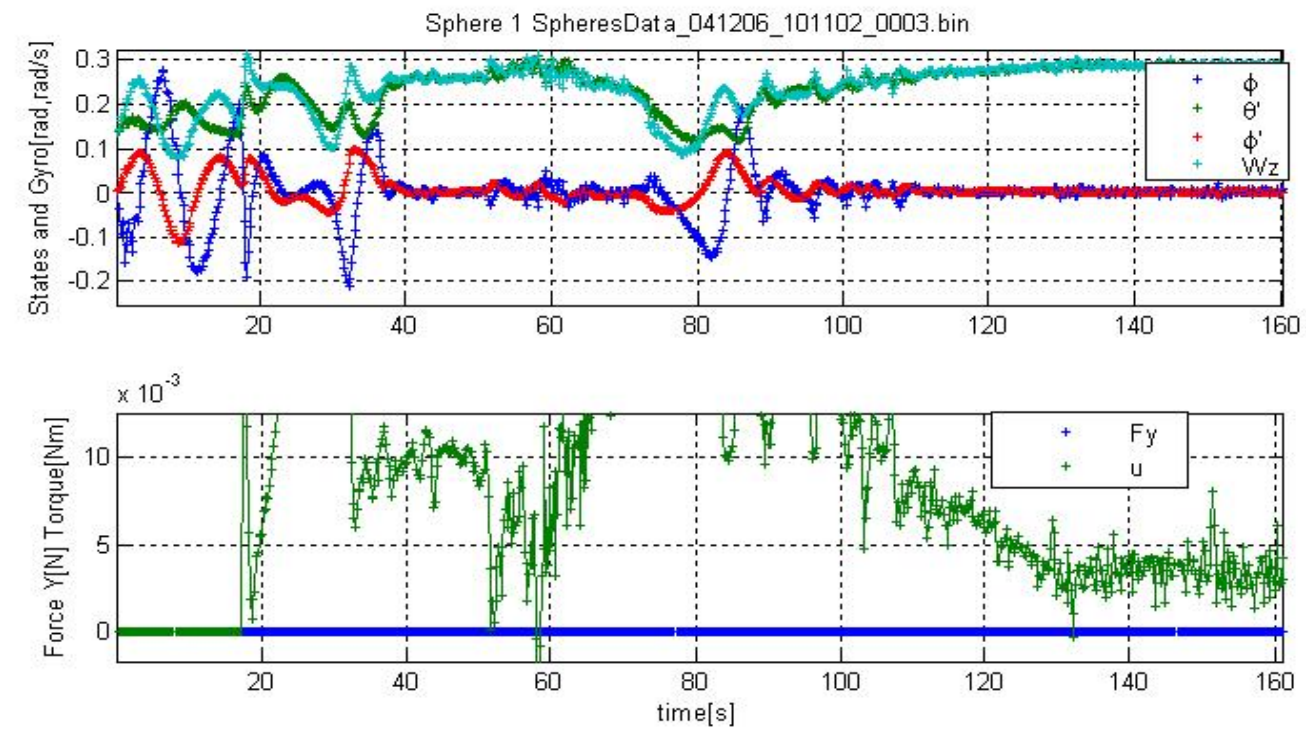

Figure 13. Gain-scheduled LQR controller in a singled-tethered system

This gain-scheduled LQR controller has been successfully implemented in the SPHERES testbed. Figure 13 shows the states background telemetry information directly recorded via the communication link from one of the satellites. The green line indicates the array angular $\operatorname{rate}(\dot{\theta})$;blue is $\phi$;red is $\dot{\phi}$;light blue is gyro data. The second row of the figure represents the actuator output (the torque has a saturation limit at $0.012 \mathrm{Nm}$ ). The LQR controller tried to spin up two tethered satellites into $0.3 \mathrm{rad} / \mathrm{s}$ from some arbitrary maneuvers. This control utilized only torque actuators to regulate the spin-up rate and to minimize the 
compound pendulum mode with the fixed tether length. The satellite encountered a sticky spot on the flat floor around 60-70 second interval, but shows a robust response back to the target angular rate. The constant torque actuation at the steady state indicates an existence of the surface friction which was not considered for linear modeling.

\section{Nonlinear Control and Experimental Results}

Even though the gain-scheduled LQR controller performed well for a regulation problem, The linear control performance can be problematic for a tracking purpose. In addition, the transient response until the states reach the steady-state is unsatisfactory - quite a large $\phi$ angle is observed in figure 13. More smooth transient responses are desired. Furthermore, we can explore the effects of un-modeled dynamics like friction more easily in a nonlinear setup. Therefore, a nonlinear controller fully accounting for the nonlinear dynamics has been developed and implemented in the SPHERES testbed.

\section{A. Feedback Linearization For Fully Actuated Systems}

A nonlinear control approach based on Input-State Feedback Linearization ${ }^{19}$ can be employed when the system is fully actuated (i.e both thruster force $F$ and torque $u$ are available as control input)

A slightly modified form of the nonlinear equation (8) is presented:

$$
\begin{aligned}
& {[M(q)](\ddot{q})+[C(q, \dot{q})](\dot{q})+[K](\dot{q})=[F]} \\
& \text { where }[M]=\left[\begin{array}{cc}
I_{G}+m r^{2}+m l^{2}+2 m r l \cos \phi & I_{G}+m r^{2}+m r l \cos \phi \\
I_{G}+m r^{2}+m r l \cos \phi & I_{G}+m r^{2}
\end{array}\right], \\
& {[C(q, \dot{q})]=\left[\begin{array}{cc}
-m r l \sin \phi \dot{\phi} & -m r l \sin \phi(\dot{\theta}+\dot{\phi}) \\
+m r l \sin \phi \dot{\theta} & 0
\end{array}\right],} \\
& {[K]=\left[\begin{array}{cc}
k_{1} & 0 \\
0 & k_{2}
\end{array}\right] \text { is the friction coefficient. and }} \\
& (q)=\left(\begin{array}{c}
\theta \\
\phi
\end{array}\right) \quad F=[M(q)] v+[C(q, \dot{q})] \dot{q}+[K](\dot{q})
\end{aligned}
$$

where $v$ is the new control input.

$$
v=\ddot{q_{d}}-2 \lambda \dot{\tilde{q}}-\lambda^{2} \tilde{q}
$$

where $\tilde{q}=q-q_{d}$. and $\lambda>0$.

If a target angular rate is $\omega$ and the objective of the control is to spin up the interferometric array to $\omega$ while minimizing the pendulum mode, a desired trajectory is defined as follows: $\dot{\theta}_{d}=\omega\left(1-e^{-\tau t}\right)+\dot{\theta}_{0}$, $\ddot{\theta}_{d}=\omega \tau e^{-\tau t}, \ddot{\phi}_{d}=0, \dot{\phi}_{d}=0, \phi_{d}=0$.

Then, Eq. (65) is re-formulated as:

$$
v=\left(\begin{array}{c}
\omega t e^{-\tau t}-\lambda\left(\dot{\theta}-\omega\left(1-e^{-\tau t}\right)-\dot{\theta}_{0}\right) \\
-2 \lambda \dot{\phi}-\lambda^{2} \phi
\end{array}\right)
$$

Note that an elimination of the rigid body mode $\theta$ resulted in a first-order differential equation as opposed to the second order for $\phi$.

This will lead to the exponentially stable closed-loop dynamics like the following:

$$
\ddot{\tilde{q}}+2 \lambda \dot{\tilde{q}}+\lambda^{2} \dot{\tilde{q}}=0
$$

Figure 14 shows the experimental data with this nonlinear controller collected from the first SPHERES of a two-body formation whereas figure 15 shows the second SPHERES tethered with the first one (The communication link for the second SPHERES somehow failed at 91 second). The third row of the figures represents the tether length measured directly using the ultrasound pseudo-GPS metrology system. It is 


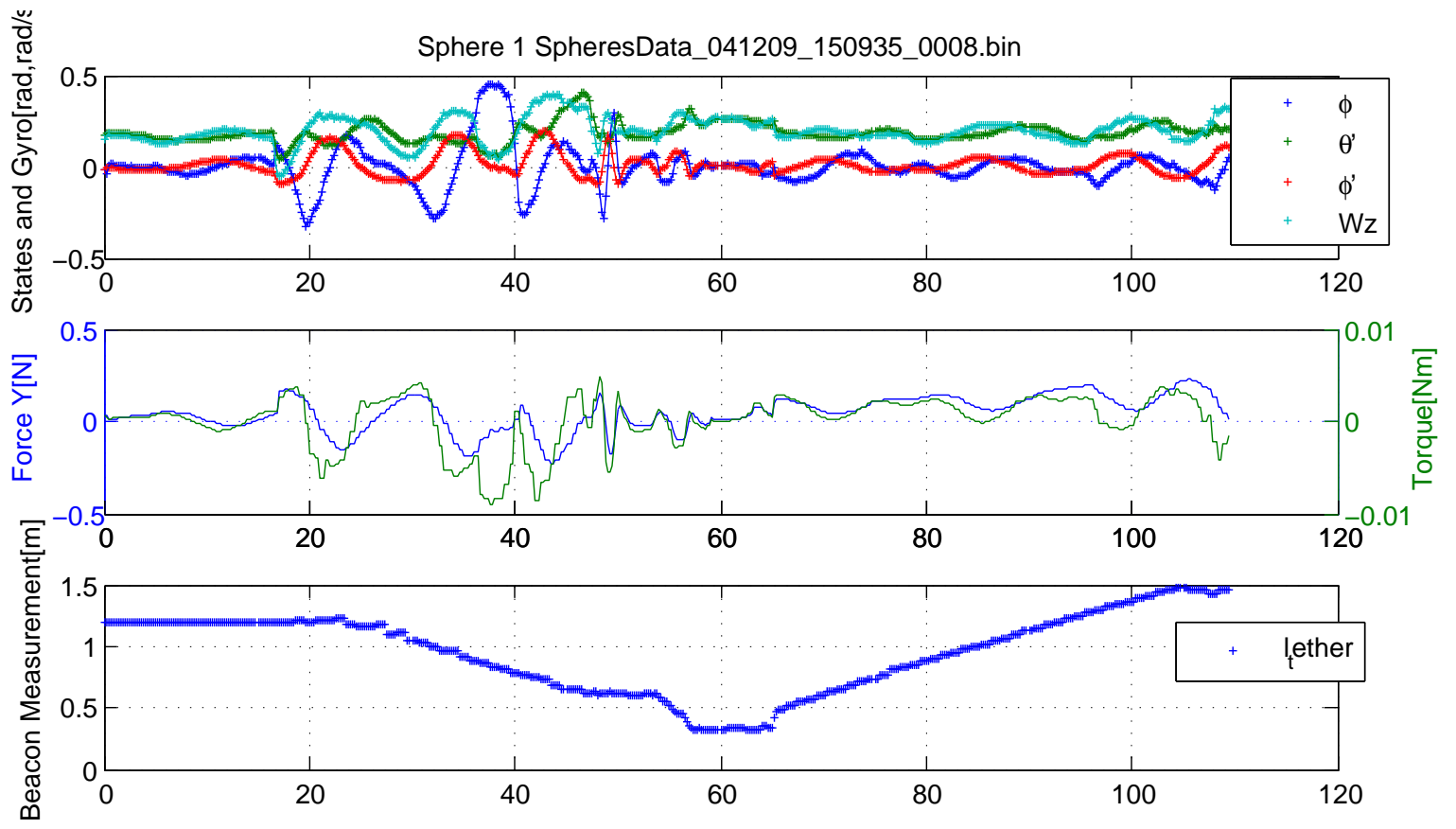

Figure 14. nonlinear controller with varying tether length in two tethered satellites, Sat1 21 of 26 


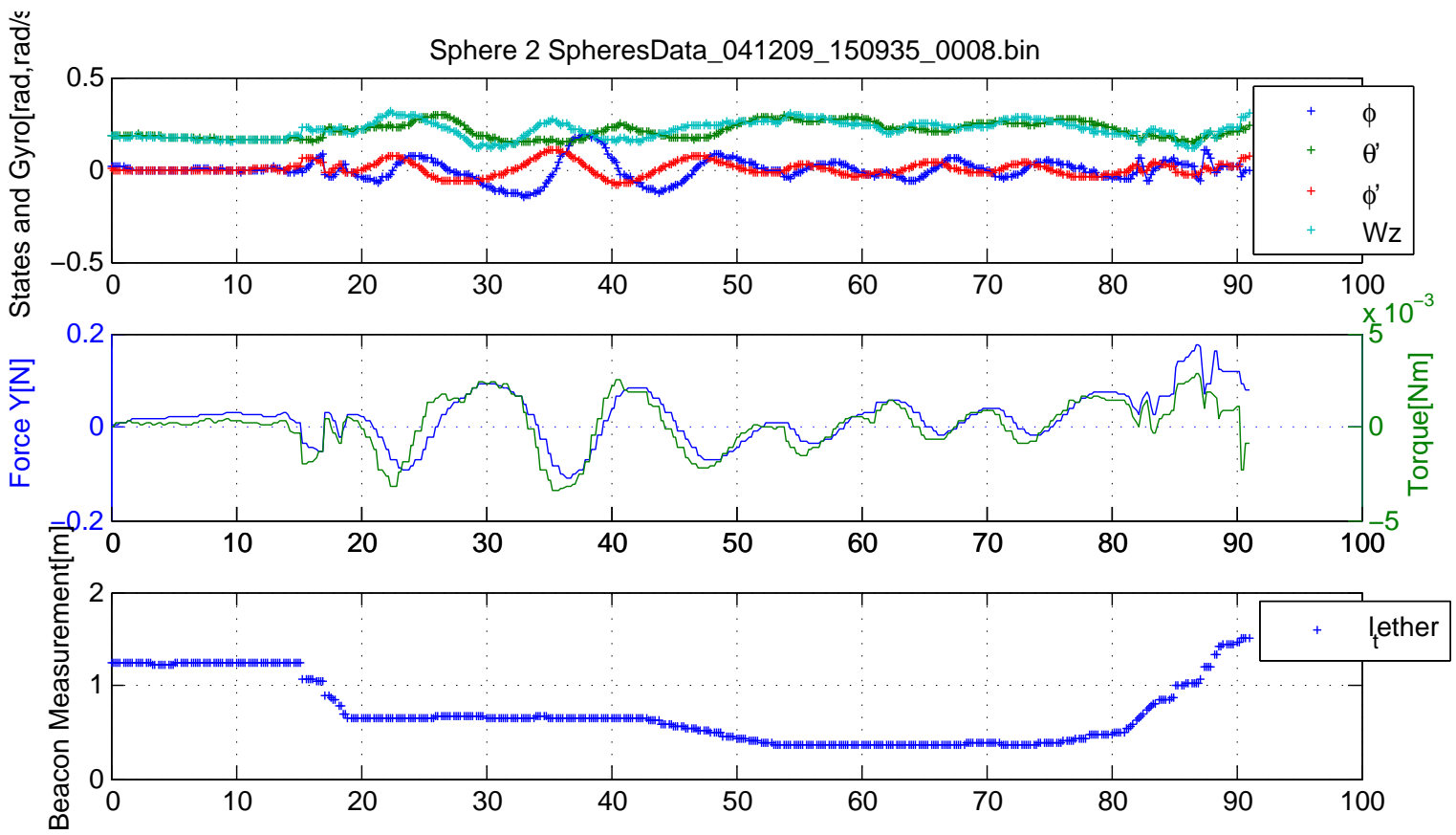

Figure 15. nonlinear controller with varying tether length in two tethered satellites, Sat2 22 of 26 
observed that the pendulum mode $(\phi, \dot{\phi})$ gets excited when the tether reels in as predicted in III-E. Note that the effect of varying the tether length, or the speed, $i$ was not considered in the nonlinear model. So better performance is achievable by taking into account the motor speed $\dot{l}$. In addition, compared to the linear controller, a more smooth transient response is accomplished. The steady state error appears somewhat larger than that of the linear control results in the previous section. This is because $1 \mathrm{~Hz}$ of control sampling rate was selected for the control while $10 \mathrm{~Hz}$ was used for the linear controller. It is a call for the designer to make to balance between reduction of steady state errors by increasing the control bandwidth, and smoothing of the high frequency disturbances by lowering the bandwidth. The experiments suggest that a lower control bandwidth like $1 \mathrm{~Hz}$ or $2 \mathrm{~Hz}$ perform well at the expense of the modest increase in steady state errors.

\section{B. Friction Modeling}

As mentioned in the previous sections, the surface friction between the flat floor surface and the air-carriage pucks is playing a significant role (see constant $F$ actuation at the steady state in figure 13). Since the nonlinear controller is based upon a precise model, investigation on the friction is indispensable to achieve satisfactory performance of the control.

Instead of the Coulomb surface friction model, a friction term proportional to the velocity is used as shown in Eq. (63). This is a reasonable assumption because there is gas flowing between the air-bearing pucks and the flat surface. The friction model was proven by comparing the Matlab-Simulink model using the nonlinear dynamics derived previously with the actual experiment data.

During the test sessions at the NASA MSFC, December 2004, lack of friction model was identified as the main problem of the initial poor performance of the nonlinear controller. After adding this friction model, the performance became satisfactory.

\section{Partial Feedback Linearization for Underactuated Systems}

The previous experiment results are based upon Full State Feedback Linearization when $F$ is available. In contrast with LTI systems in which underactuated controller can be implemented easily, it is difficult to meet the goal of designing a nonlinear controller for nonlinear underactuated systems. This is because an underactuated nonlinear system like single tethered spacecraft with torque actuators only $(F=0, u \neq 0)$ is NOT Full State Feedback Linearizable. Underactuated mechanical systems are characterized by fewer actuators than degree of freedom. Common 2 DOF examples include pendubot and acrobot where input is available only to one variable. From Eq. (8), this single tethered system is underactuated via input coupling. The preliminary result of developing nonlinear underactuated controllers is presented.

Partial feedback linearization ${ }^{23,24}$ technique is applied to Eq. (6),

$$
\begin{aligned}
& m_{11} \ddot{\theta}+m_{12} \ddot{\phi}+h_{1}=0 \\
& m_{21} \ddot{\theta}+m_{22} \ddot{\phi}+h_{2}=u
\end{aligned}
$$

where $m_{11}=m l+m r \cos \phi, m_{12}=m r \cos \phi, m_{21}=I_{G}+m r^{2}+m r l \cos \phi, m_{22}=I_{G}+m r^{2}$, $h_{1}=-m r \sin \phi(\dot{\theta}+\dot{\phi})^{2}$, and $h_{2}=m r l \sin \phi \dot{\theta}^{2}$

Note the first equation of the system is a second order nonholonomic constraint. This system is feedback linearized partially for $\phi$ like the following procedures,

$$
\begin{gathered}
\ddot{\theta}=-m_{11}{ }^{-1} m_{12} \ddot{\phi}-m_{11}{ }^{-1} h_{1} \\
\ddot{\phi}=v \\
u=\alpha(\phi) v+\beta(\phi, \dot{\theta}, \dot{\phi}) \\
\alpha(\phi)=m_{22}-m_{21} m_{11}{ }^{-1} m_{12} \\
\beta(\phi, \dot{\theta}, \dot{\phi})=h_{2}-m_{21} m_{11}{ }^{-1} h_{1}
\end{gathered}
$$

where $v$ is now a new control input to $\phi$ dynamics. 
Then, we can design a controller $v$ to asymptotically stabilize $\phi$ dynamics. To investigate the trajectory of $\theta$ under this control input, $\theta$ dynamics in Eq. (70) is expanded to:

$$
\begin{gathered}
\ddot{\theta}=-m_{11}{ }^{-1} m_{12} \ddot{\phi}-m_{11}{ }^{-1} h_{1} \\
=-m_{11}{ }^{-1}\left(m_{12} v+h_{1}\right) \\
=-m_{11}{ }^{-1}\left((m r \cos \phi) v-m r \sin \phi(\dot{\theta}+\dot{\phi})^{2}\right) \\
=-m_{11}{ }^{-1} m r \cos \phi\left(v-\tan \phi(\dot{\theta}+\dot{\phi})^{2}\right)
\end{gathered}
$$

Since $m_{11}$ is a function of $\phi$ only, the righthand side of Eq. (71) is bounded as long as:

$$
\begin{gathered}
l>r,|\phi|<\pi / 2, \text { and } \phi, \dot{\phi} \rightarrow 0 \\
v=\tan \phi(\dot{\theta}+\dot{\phi})^{2}+v_{\text {new }}
\end{gathered}
$$

The following nonlinear controller is proposed for single tethered underactuated systems.

$$
\begin{gathered}
v=\tan \phi(\dot{\theta}+\dot{\phi})^{2}-K_{\phi} \phi-D_{\phi} \dot{\phi}+D_{\theta}\left(\dot{\theta}_{r e f}-\dot{\theta}\right) \\
u=\alpha(\phi) v+\beta(\phi, \dot{\theta}, \dot{\phi})
\end{gathered}
$$

where $K, D$ and $D_{\theta}$ are real positive numbers.

The performance of such controller for a single tethered SPHERES rotating initially at $0.3 \mathrm{rad} / \mathrm{s}$ with 1 $\mathrm{m}$ tether length is simulated (see figure 16). This controller is superior to a linear underactuated controller in section VI in terms of smoothness of transient response while fully accounting for nonlinear dynamics of the system. This sort of controllers using partial linearization technique have yet to be implemented in the SPHERES testbed in the near future.
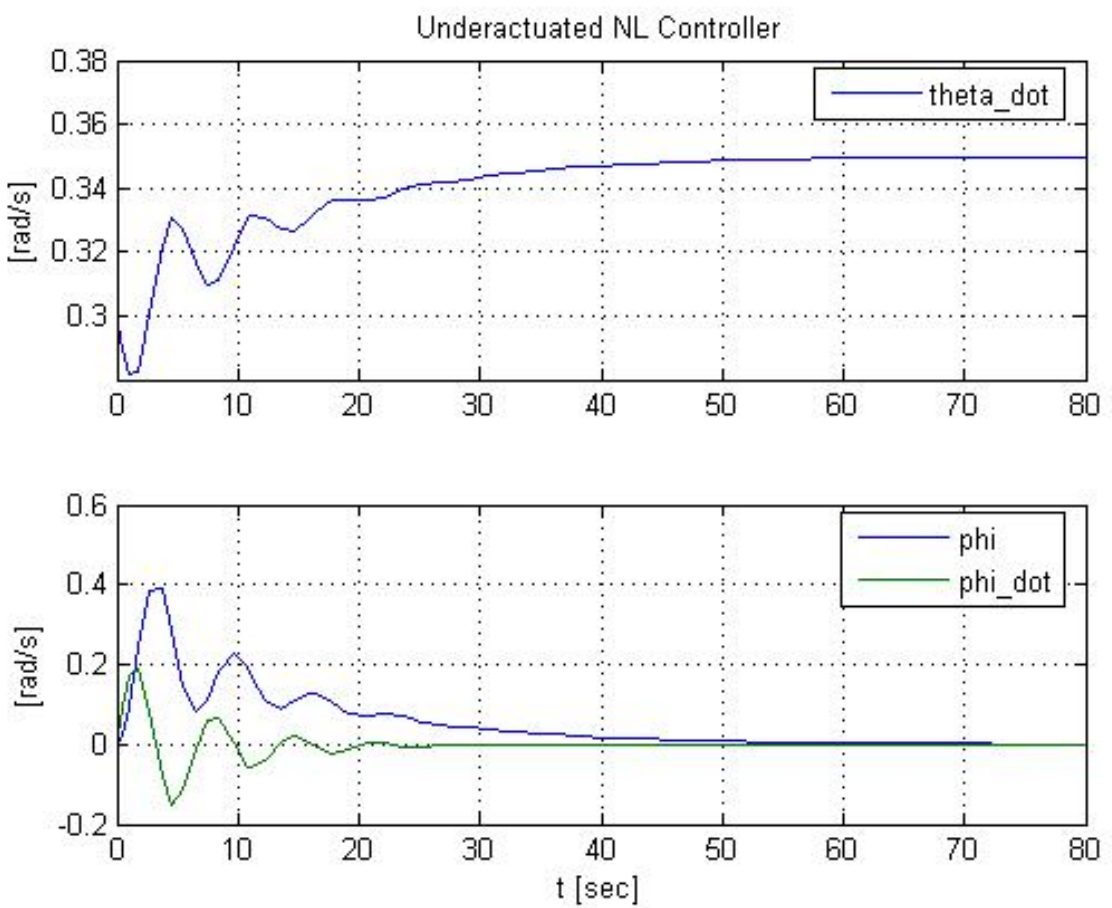

Figure 16. Performance of underactuated controller $l=1 \mathrm{~m}, \dot{\theta}_{0}=0.3$ and target $\dot{\theta}=0.35 \mathrm{rad} / \mathrm{s}$ 


\section{Conclusion}

The objective of the tethered SPHERES project is to demonstrate the feasibility of controlling array formation without thrusters for the future space interferometer missions. Another goal is to develop sophisticated decentralized control and estimation algorithms for the tethered system eliminating the need for satellite communications. The decentralized scheme is realized by the decoupling technique and contraction theory. It is proven that a decentralized control law can be designed from the single tethered system in lieu of the two spacecraft dynamics reducing the computation and communication burdens. The controllability analysis indicates that both array resizing and spin-up are fully controllable only by the reaction wheels and the tether motor, thereby eliminating the need for thrusters. Linear and nonlinear decentralized control techniques have been implemented into the tethered SPHERES testbed, and tested at the NASA MSFC's flat floor facility using two and three SPHERES configurations. The performance of the LQR controller gain-scheduled over the operating angular rate using only reaction wheels was sometimes unsatisfactory resulting in a large pendulum mode angle. The nonlinear control using feedback linearization technique performed successfully in both two SPHERES in-line configuration and three triangular configuration while varying the tether length. The relative sensing system, using the ultrasound ranging system and the inertial sensors as well as the decentralized nonlinear estimator, is developed to provide necessary state information. We are currently updating the testbed with RWA and more sophisticated bearing angle measurement system with force-torque sensor. The video clips of the experiments in this paper can be downloaded at http://ssl.mit.edu/spheres/videos.html.

\section{Acknowledgments}

The authors would like to gratefully acknowledge NASA for both financial and technical support for the MIT-SSL and PSI SPHERES Tether program. This work has been sponsored under NASA Phase II SBIR contract (Contract No.: NNG05CA09C). The authors would like to gratefully appreicate the technical support from the MIT SPHERES team including Steve Sell, Alvar Otero, Mark Hilstad, Simon Nolet, and Danielle Adams.

\section{References}

\footnotetext{
${ }^{1}$ Chung, S.-J., Miller, D.W., deWeck, O.L., "ARGOS Testbed: Study of Multidisciplinary Challenges of Future Spaceborne Interferometric Arrays," SPIE Optical Engineering, Vol. 43, No. 9, pages 2156-2167, September 2004.

${ }^{2}$ Beichman, C.A., Woolf, N.J., and Lindensmith, C.A., "The Terrestrial Planet Finder (TPF): A NASA Origins Program to Search for Habitable Planets," JPL Publication 99-3, 1999.

${ }^{3}$ Mather, J.C et al., "The Submillimeter Frontier: A Space Science Imperative," Astrophysics 1998, astro-ph/9812454.

${ }^{4}$ Belanger, G. M., et al., "Decentralized Control of Satellite Clusters Under Limited Communication," AIAA 2004-5023, AIAA GNC Conference, Providence, RI, 2004.

${ }^{5}$ Smith, R. S., and Hadaegh, F. Y., "Control of Deep-Space Formation-Flying Spacecraft; Relative Sensing and Switched Information," AIAA J. GCD, Vol. 28, No. 1 Jan-Feb 2005.

${ }^{6}$ Kong, M.C., et al, "SPHERES as a Formation Flight Algorithm Development and Validation Testbed: Current Progress and Beyond," 2nd International Symposium on Formation Flying Missions and Technologies, September 14-16, 2004, Washington, DC.

${ }^{7}$ Wilson, J.M., Control of a Tethered Artificial Gravity Spacecraft, Ph.D. thesis, Stanford University, 1990.

${ }^{8}$ Beletsky and Levin, Dynamics of Space Tether Systems, American Astronautical Society Publication Vol. 83.

${ }^{9}$ Nakaya, K, et al., "On Formation Deployment for Spinning Tethered Formation Flying and Experimental Demonstration," 18th International Syposium on Space Flight Dynamics, 11-15 October 2004, Munich, Germany.

${ }^{10} \mathrm{Yu}$, S., "Dynamic Model and Control of Mass-Distributed Tether Satellite System," J. Spacecraft and Rockets, Vol.39 No.2, March-April 2002.

${ }^{11}$ Kojima, H, et al, "Nonlinear Control of Librational Motion of Tethered Satellites in Elliptic Orbits," J of Guidance, Control and Dynamics, Vol.27, No. 2, March-April 2004.

${ }^{12}$ Kokubun, K, et al, "Deployment,Retrieval Control of Tethered Subsatellite Under Effect of Tether Elasticity," J. of Guidance, Control and Dynamics, Vol.19,No.1, January-February 1996.

${ }^{13}$ Fujii, H.A., "Wave-Absorbing Control of Transverse Vibration of Tethered System," Journal of Astronautical Sciences, Vol 51, No.3, pp 249-259, 2004.

${ }^{14}$ Kim, M., and Hall, C.D., "Dynamics and Control of Tethered Satellite Systems for NASA's SPECS Mission," AAS03-532.

${ }^{15} \mathrm{Kim}$, M., and Hall, C.D., "Control of a Rotating Variable-Length Tethered System," J. of Guidance, Control and Dynamics, Vol.27, No.5, Sep-Oct 2004.

${ }^{16}$ Bombardelli, C., Lorenzini, E.C., Quadrelli, M. B., "Retargeting Dynamics of a Linear Tethered Interferometer," AIAA J. of Guidance, Control and Dynamics, Vol. 27, No.6, Nov-Dec 2004.
} 
${ }^{17}$ Kumar, K.D. and Yasaka, Y., "Rotating Formation Flying of Three Satellites Using Tethers," J. Spacecraft and Rockets, Vol. 41, No. 6, Nov-Dec 2004.

${ }^{18}$ Nolet, S., Kong, E., and Miller, D.W., "Autonomous docking algorithm development and experimentation using the SPHERES testbed," SPIE Defend and Security Symposium, April 2004, Orlando, FL.

${ }^{19}$ Slotine and Li, Applied Nonlinear Control, Prentice Hall,. New Jersey, 1991.

${ }^{20}$ Lohmiller, W., and Slotine, J.-J E., "On Contraction Analysis for Nonlinear Systems," Automatica, 34(6), 1998.

${ }^{21}$ Slotine, J.-J E., "Modular Stability Tools for Distributed Computation and Control," Int. J. Adaptive Control and Signal Processing, 17(6), 2003.

${ }^{22}$ Wang, W., and Slotine, J.J.E., "On Partial Contraction Analysis for Coupled Nonlinear Oscillators," Biological Cybernetics, 92(1), 2004.

${ }^{23}$ Spong, M. W., "The Swing Up Control Problem For The Acrobot." IEEE Control Systems, pp. 49-55, February 1995.

${ }^{24}$ Olfati-Saber, R. "Nonlinear Control of Underactuated Mechanical Systems with Application to Robotics and Aerospace Vehicles," Ph.D thesis, Department of EECS, MIT, February 2001. 